\title{
Feature-oriented joint time-lapse seismic and electromagnetic history matching using ensemble methods
}

\author{
Yanhui Zhang, Ibrahim Hoteit \\ King Abdullah University of Science and Technology
}

\begin{abstract}
We propose a feature-oriented ensemble history matching workflow with a focus on the integration of time-lapse seismic and electromagnetic (EM) data. The developed workflow consists of two main steps. Firstly, the rock cross-properties such as water saturation and porosity relating to both seismic and EM attributes are estimated by joint inversion of seismic and EM data using an iterative ensemble smoother. Secondly, the remaining model parameters of interest such as permeability are calibrated based on the updated crossproperties. To assimilate the inverted saturation information efficiently, we take a featureoriented integration approach in which front positions are identified from the inverted saturation field. Related model parameters are then conditioned to the interpreted fronts using the iterative ensemble smoother with a distance parameterization. The novelty of the proposed approach consists in combining the feature-oriented history matching with ensemble-based geophysical inversion to achieve an efficient joint integration of multiple sources of geophysical data. The performance of the proposed history-matching workflow is examined using a 2D channelized reservoir model and a more realistic 3D reservoir model with a crosswell configuration for seismic and EM surveys. It is demonstrated that the developed workflow provides a novel and effective way to calibrate reservoir models with multiple sources of geophysical data. The experimental results show a positive synergy effect on the characterization of model variables by jointly assimilating seismic and EM data.
\end{abstract}




\section{Introduction}

Joint inversion of multiple sources of geophysical data has been actively studied in the geophysical community $[22,37,2,31,53,36,48,10]$, due to its great potential to enhance the fidelity of inverted models. The core idea of joint inversion is to exploit the complementary nature between different types of geophysical data sets, and combine the information into an integrated inversion scheme for better recovery of properties or structures within the reservoir. It is well known that seismic and electromagnetic (EM) data inherently exhibit complementary sensitivities to reservoir rock and fluid properties and are increasingly used for joint inversion [19, 22]. Generally, seismic measurements provide effective information for reservoir structure while electromagnetic measurements are sensitive to fluid content for distinguishing between oil and water. Dell'Aversana et al. [11] proposed a joint inversion of the so-called rock cross-properties (e.g., porosity and fluid saturation) from different types of well-log measurements. Because these cross-properties provide a common link to different measurements, they showed that it is possible to reduce the interpretative ambiguities of these properties through joint inversion. Gao et al. [16] introduced a joint petrophysical inversion approach integrating EM and full-waveform seismic data. Their results showed that the joint inversion enhanced the characterization of porosity and water saturation maps when the petrophysical relationships therein were reasonably quantified.

While the gradient-based optimization using the adjoint method is dominantly applied for geophysical inversion, the ensemble-based assimilation methods (for reviews, see e.g., $[1,43])$, such as the ensemble Kalman filter (EnKF) and the ensemble smoother (ES), are increasingly used for solving history-matching problems in the reservoir engineering community. As summarized by Oliver and Chen [43], in contrast to the adjoint-based or other history matching methods, the ensemble-based methods have the advantages of easy adaptability to different data types and model variables (even in high dimensions), efficient parallelizable implementation, and quantitative assessment of uncertainty (even though generally underestimated). Significant research has been done to enhance the performance of the standard EnKF or ES in dealing with realistic problems characterized 
by high non-linearity and non-Gaussianity [25, 58, 46, 20, 32, 8, 13, 59, 21, 3, 9, 44].

Distinct from the assimilation of production data that are commonly used for history matching, integrating geophysical measurements using ensemble methods poses new challenges [57]. Firstly, the number of geophysical data is generally very large, however, the limited ensemble size (typically chosen in practice for computational efficiency and feasibility) severely constrains the ability of the ensemble-based methods to match large amounts of accurate and independent data. Even though there are a number of general mitigation techniques (e.g., localization [7] and inflation [30]) available, each of them has its own drawbacks limiting its efficiency in real applications [29]. Some recent research has been focused on seeking a sparse representation efficiently reducing the number of original data while still capturing the essential information contained in the data $[54,40,33,57]$. Secondly, the approach to assimilate geophysical data, such as seismic and EM data measurements, is not unique [50, 60]. There are three different levels at which the extracted information from the raw data can be utilized for updating reservoir models, as shown in Fig. 1. Each level involves different degrees of forward modeling and inversion processes. In general, no matter which level is selected, obtaining a consistent comparison between measured (including interpreted or inverted geophysical attributes) and simulated data is not trivial. There is a growing investigation of incorporating different forms of geophysical data and studying their performances under the ensemble-based history-matching framework $[51,15,30,27,60]$. Lastly, geophysical data involve large uncertainty that stems from the whole acquisition-processing-interpretation process and is usually difficult to quantify. In addition, the perplexing physical conditions and processes associated with these measurements hamper the construction of representative forward models predicting the reality reliably. Oliver and Alfonzo [41] proposed a systematic approach to deal with the problem of assimilating biased and inaccurate observations with imperfect models, in which an iterative procedure involving model criticism and improvement is introduced following the process of model creation and calibration. The methodology was further applied to assimilate seismic data under a synthetic experimental setting, showing reduced tendency for overfitting and improved predictability [4]. 


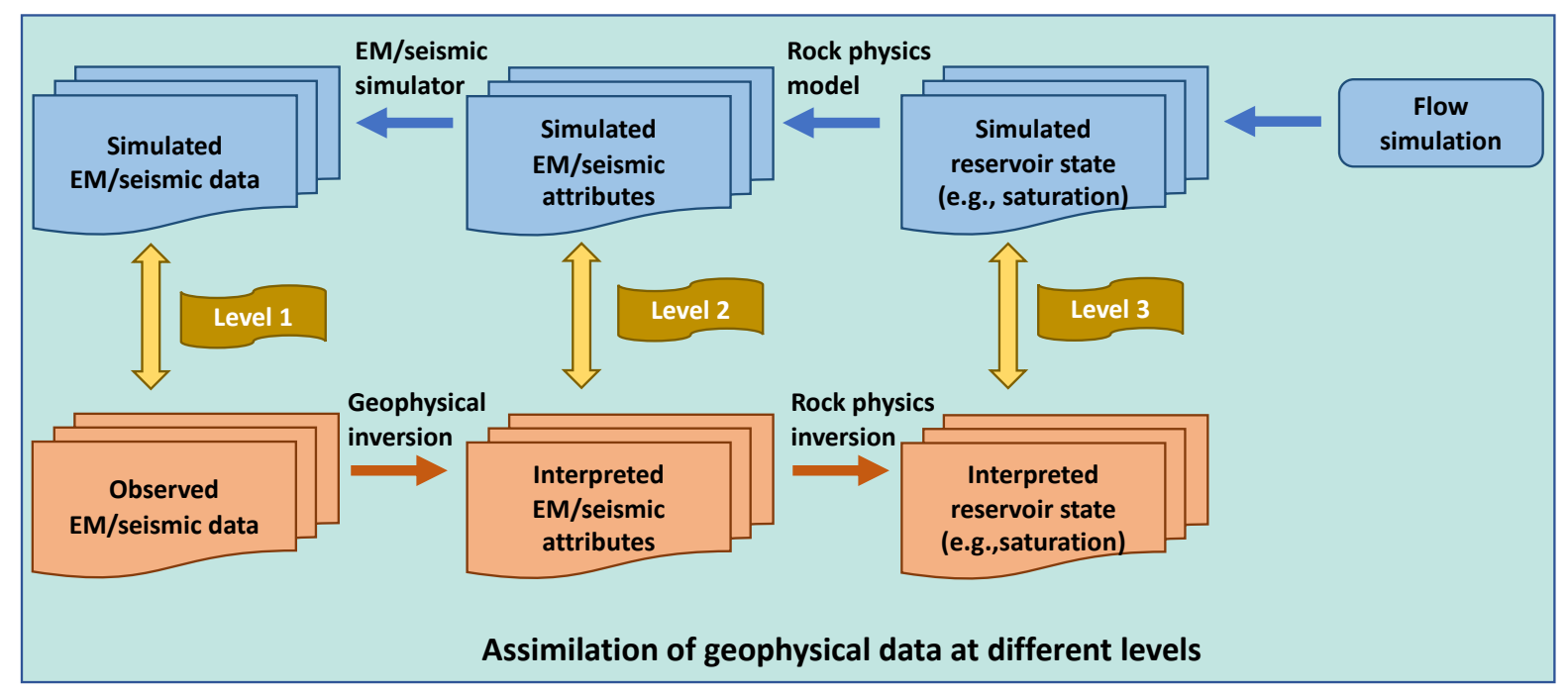

Fig. 1: Schematic diagram for the integration of geophysical measurements in different ways.

Recently, Zhang et al. [60] examined the integration of crosswell EM data from the first two levels (as in Fig. 1), which correspond to the conditioning on raw EM observations (after necessary data processing) at Level 1 and interpreted conductivity through geophysical inversion at Level 2, respectively. Instead of integrating the inverted conductivity directly, they updated uncertain model variables using the features or patterns (i.e., the boundary of conductivity contrast) detected from the inverted conductivity field. Because the detected features typically provide a sparse representation of original data while still capturing the essential information, the feature-oriented integration approach turned out to be more efficient than the direct integration of inverted conductivity. Zhang et al. [60] showed that both levels gave comparable history matching results under relatively simple experimental settings. For Level 2, however, the performance of the feature-oriented integration approach somehow depended on the quality of inversion results and was susceptible to the biases and errors introduced by the inversion. In the case that multiple sources of geophysical data are available, implementing the feature-oriented integration at Level 3 seems preferable over Level 2. The reason is at least twofold. First, Level 3 provides an opportunity for joint inversion, from which reservoir cross-properties linked to different geophysical attributes (e.g., seismic velocities and formation conductivity) are inverted. Therefore, compared with the separate inversion at Level 2, the joint inversion 
at Level 3 holds the potential to enhance the performance of the feature-oriented integration approach. Second, it is more efficient to assimilate the features extracted from the inverted cross-property which synthesizes the information of different geophysical attributes at Level 2. As an extension of our previous work [60], we propose a featureoriented history-matching workflow focusing on the joint integration of time-lapse seismic and EM data in this study. The novelty of the proposed method consists in combining the feature-based history matching with ensemble-based geophysical joint inversion for enhanced model calibration.

In the remainder of this paper, we first give an overview of the developed featureoriented ensemble history-matching workflow, then explain the major components of the methodology in detail, followed by numerical experiments in which we examine the performance of the proposed method, and demonstrate the benefit of conditioning reservoir models to both seismic and EM data.

\section{Methodology}

\subsection{Feature-oriented ensemble history-matching workflow}

History matching is a well-known type of inverse problem in reservoir modeling. Given the relationship between the model variables $\mathbf{m} \in \Re^{N_{m}}$ and the observable variables $\mathbf{d}_{o b s} \in \Re^{N_{d}}$, denoted by

$$
\mathbf{d}_{o b s}=\mathbf{g}(\mathbf{m})+\varepsilon_{d}
$$

where $\varepsilon_{d}$ represents observation error, the inverse problem seeks for $\mathbf{m}$ that is the solution of Eq. (1) given $\mathbf{d}_{\text {obs }}$. The forward operator $\mathbf{g}(\cdot)$ represents a model of the true physical relation between reservoir properties and data. In a Bayesian framework, model parameters are considered as random variables and their distributions are described with probability density functions (PDF). According to Bayes' theorem, the posterior PDF of $p\left(\mathbf{m} \mid \mathbf{d}_{o b s}\right)$ for model variables $\mathbf{m}$ given observations $\mathbf{d}_{o b s}$ is defined as

$$
p\left(\mathbf{m} \mid \mathbf{d}_{o b s}\right) \propto p\left(\mathbf{d}_{o b s} \mid \mathbf{m}\right) p(\mathbf{m})
$$


where the normalizing constant in the denominator is omitted and hence the equal sign is replaced with the proportional symbol [6]. In Eq. (2), the prior PDF of $p(\mathbf{m})$ represents the uncertainty of model parameters before incorporating the observations. It describes the dependency between model parameters and imposes constraints on the set of possible solutions. The conditional PDF of $p\left(\mathbf{d}_{o b s} \mid \mathbf{m}\right)$, known as the likelihood function, quantifies the likelihood of observing $\mathbf{d}_{o b s}$ given $\mathbf{m}$. In this formulation, the solution to the aforementioned inverse problem (or history matching) is given by the posterior PDF, which is proportional to the product of the prior PDF and the likelihood function.

Without loss of generality, we assume that the model variables $\mathbf{m}$, customarily organized in the form of a vector, include reservoir porosity $\phi$ and permeability $\kappa$. The extension to include other types of model variables is straightforward. Regarding the observations $\mathbf{d}_{o b s}$, we focus on time-lapse seismic and EM data. Compared with the commonly used production data (water, oil, and gas rates and pressures), seismic and EM data involve more complex forward-modeling processes and possess much higher spatial resolutions. To estimate the posterior distribution of the model parameters of interest given the data, we take a two-step integration approach as

$$
p\left(\kappa, \phi \mid \mathbf{d}_{o b s}^{S e i s+E M}\right)=\int p(\kappa \mid \phi, S) p\left(\phi, S \mid \mathbf{d}_{o b s}^{S e i s+E M}\right) \mathrm{d} S,
$$

where $S$ represents reservoir water saturation at a certain time (time index is ignored for concision). Here, we assume that $\kappa$ depends only on $\phi$ and $S$. It is generally impractical to evaluate the entire posterior PDF of model variables. A feasible way to explore the posterior PDF is by sampling. In the first step, we sample the realizations of the so-called rock cross-properties [11] such as $\phi$ and $S$ from the posterior PDF of $p\left(\phi, S \mid \mathbf{d}_{o b s}^{\text {Seis+EM }}\right)$. Because these cross-properties relate to both seismic and EM attributes, it provides a way for joint geophysical inversion. In the second step, we sample the realizations of the remaining model parameters such as $\kappa$ from the conditional PDF of $p(\kappa \mid \phi, S)$ based on the sampled cross-properties.

Step 1: ensemble-based joint inversion. Applying Bayes' theorem as in Eq. (2) 
to $p\left(\phi, S \mid \mathbf{d}_{\text {obs }}^{\text {Seis }+E M}\right)$, we obtain

$$
p\left(\phi, S \mid \mathbf{d}_{o b s}^{\text {Seis }+E M}\right) \propto p\left(\mathbf{d}_{o b s}^{\text {Seis }+E M} \mid \phi, S\right) p(\phi, S)
$$

where $p(\phi, S)$ denotes the prior PDF of $\phi$ and $S$. To evaluate the likelihood function $p\left(\mathbf{d}_{\text {obs }}^{\text {Seis }+E M} \mid \phi, S\right)$, we need the forward model that links reservoir porosity and saturation to seismic and EM observations. Rock-physics modeling [35] is first needed to build direct or indirect relations between the petrophysical properties and the seismic and EM attributes such as seismic velocity $V$ and formation conductivity $\sigma$,

$$
\left[\begin{array}{l}
V \\
\sigma
\end{array}\right]=\left[\begin{array}{l}
\mathbf{f}_{v}(\phi, S)+\varepsilon_{v} \\
\mathbf{f}_{\sigma}(\phi, S)+\varepsilon_{\sigma}
\end{array}\right]
$$

where $\mathbf{f}_{v}(\cdot)$ and $\mathbf{f}_{\sigma}(\cdot)$ represent the rock-physics models for seismic velocity and formation conductivity, respectively. The error terms $\varepsilon_{v}$ and $\varepsilon_{\sigma}$ are ignored here so that deterministic rock-physics models from well log and core analysis are used. Then seismic and EM responses are modeled as

$$
\left[\begin{array}{l}
\mathbf{d}^{\text {Seis }} \\
\mathbf{d}^{E M}
\end{array}\right]=\left[\begin{array}{l}
\mathbf{h}_{s}(V)+\varepsilon_{s} \\
\mathbf{h}_{e}(\sigma)+\varepsilon_{e}
\end{array}\right]=\left[\begin{array}{l}
\mathbf{h}_{s}\left[\mathbf{f}_{v}(\phi, S)\right]+\varepsilon_{s} \\
\mathbf{h}_{e}\left[\mathbf{f}_{\sigma}(\phi, S)\right]+\varepsilon_{e}
\end{array}\right],
$$

where $\mathbf{h}_{s}(\cdot)$ and $\mathbf{h}_{e}(\cdot)$ denote the seismic and EM forward simulators, respectively. The details about the forward models $\mathbf{f}_{v}, \mathbf{f}_{\sigma}, \mathbf{h}_{s}$, and $\mathbf{h}_{e}$ used in this paper are provided in Appendices A and B.

Under the Gaussian assumptions on the prior $p(\phi, S)$ and the observation errors $\varepsilon_{s}$ and $\varepsilon_{e}$, we can apply the ensemble-based assimilation methods to sample the realizations of $\phi^{a}$ and $S^{a}$ from the posterior PDF of $p\left(\phi, S \mid \mathbf{d}_{o b s}^{S e i s+E M}\right)$. A detailed derivation of the probabilistic formulation of the ensemble-based assimilation methods can be found in $[1,14,3]$.

Step 2: feature-oriented ensemble update. Once the posterior realizations of the cross-properties $\phi^{a}$ and $S^{a}$ are sampled from Step 1, one can compute $p\left(\kappa \mid \phi^{a}, S^{a}\right)$ in the same way, as

$$
p\left(\kappa \mid \phi^{a}, S^{a}\right) \propto p\left(S^{a} \mid \phi^{a}, \kappa\right) p\left(\phi^{a} \mid \kappa\right) p(\kappa) .
$$


Expression 7 indicates a sequential updating of $\kappa$ based on $\phi^{a}$ and $S^{a}$. If we assume that $\kappa$ and $\phi$ are jointly Gaussian and their cross-correlations can be well captured based on the prior information, one can sample the realizations of $\kappa^{a, \phi}$ first based on $\phi^{a}$,

$$
p\left(\phi^{a} \mid \kappa\right) p(\kappa)=\int p\left(\phi^{a} \mid \kappa, \phi\right) p(\kappa, \phi) \mathrm{d} \phi
$$

using the ensemble-based assimilation methods, where $p(\kappa, \phi)$ represents the prior PDF of $\kappa$ and $\phi$. However, the true relationship of dependence between $\kappa$ and $\phi$ is usually poorly known from the prior information $[12,18]$. In such cases, spurious correlations and biases can be introduced into the realizations of $\kappa^{a, \phi}$. As an alternative, one can assume that $\kappa$ and $\phi$ are independent so that the conditioning on the updated $\phi$ is avoided. The drawback of this is that the true correlations between $\kappa$ and $\phi$ captured by the initial ensemble may be lost.

Instead of updating the permeability with $S^{a}$ directly, we take a feature-oriented approach by conditioning the permeability to the extracted saturation fronts from $S^{a}$, denoted by $S_{c}^{a}$. Compared with the direct assimilation of $S^{a}$, the advantages of the featureoriented approach are at least twofold. On the one hand, the information contained in the shape or outline of a feature is generally more reliable and informative than the one contained in the amplitude of individual grid cell of $S^{a}$. On the other hand, higher computational efficiency can possibly be achieved since the dimensions of the feature space are typically much lower than the original data space. Therefore, we replace $p\left(S^{a} \mid \phi^{a}, \kappa\right)$ with $p\left(S_{c}^{a} \mid \phi^{a}, \kappa\right)$, which is evaluated based on the following forward model,

$$
S_{c}=\mathbf{f}_{c}\left[\mathbf{h}_{f}\left(\phi^{a}, \kappa\right)\right]+\varepsilon_{c}
$$

where $\mathbf{h}_{f}(\cdot)$ denotes the reservoir flow simulator that links porosity and permeability to water saturation, and $\mathbf{f}_{c}(\cdot)$ represents the image processing operator that extracts the front positions from the saturation field. Note that the forward model of $\mathbf{h}_{f}$ is assumed to be perfect without modeling error here. The data error $\varepsilon_{c}$ is assumed to be Gaussian. Finally, we can sample the posterior realizations of $\kappa^{a}$ from

$$
p\left(\kappa \mid \phi^{a}, S_{c}^{a}\right) \propto p\left(S_{c}^{a} \mid \phi^{a}, \kappa^{a, \phi}\right) p\left(\phi^{a}, \kappa^{a, \phi}\right)
$$


using the ensemble-based assimilation methods. The whole history-matching workflow described above is summarized in Fig. 2.

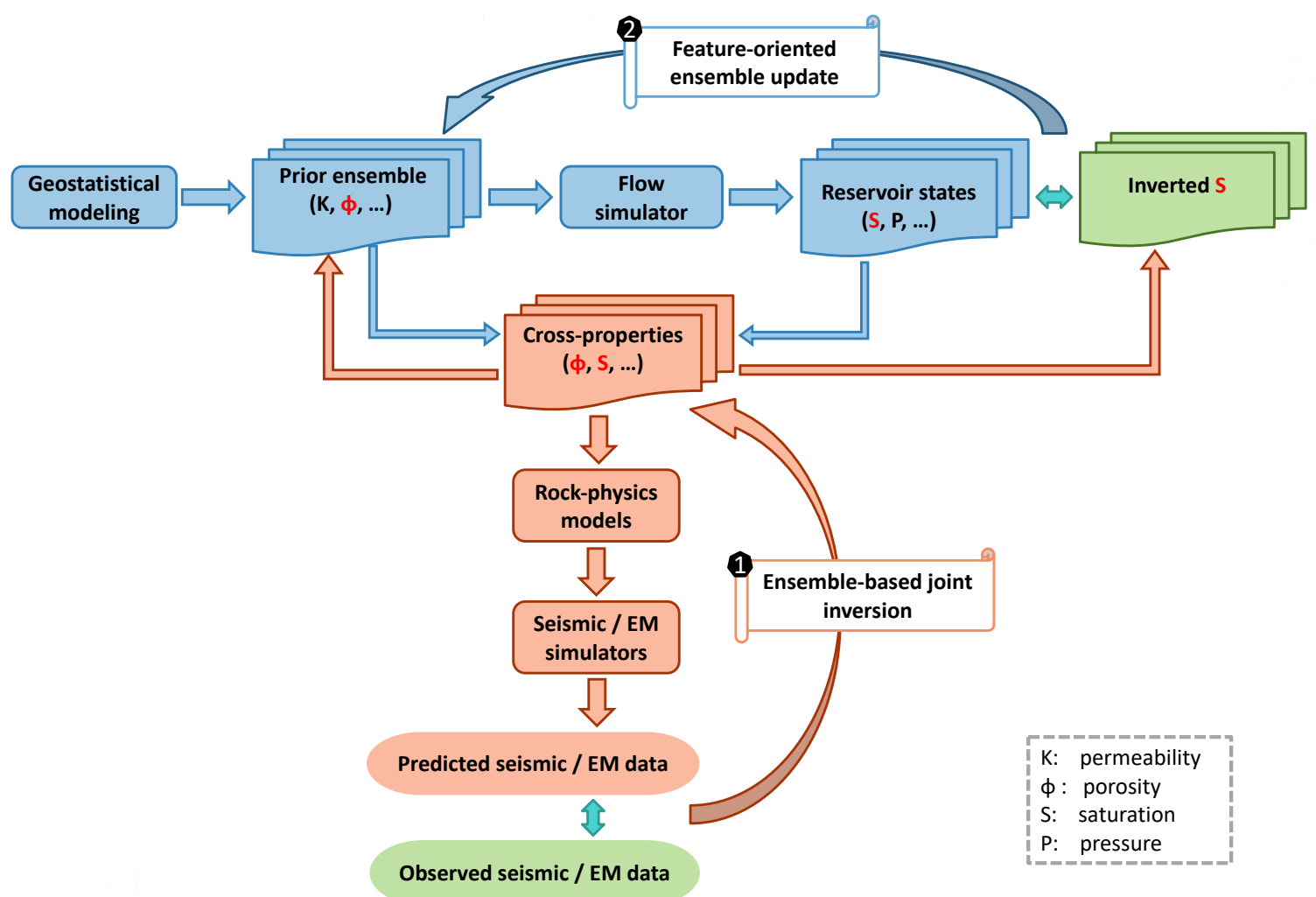

Fig. 2: Feature-oriented ensemble history-matching workflow for assimilation of time-lapse seismic and EM data.

In the following, we present the details of the methodology, focusing on the major components of the developed history-matching workflow.

\subsection{Sequential assimilation of time-lapse seismic and EM data}

Let $\mathbf{d}_{o b s, 1: n}^{S e i s+E M}=\left\{\mathbf{d}_{o b s, 1}^{S e i s+E M}, \cdots, \mathbf{d}_{o b s, n}^{S e i s+E M}\right\}$ denote the seismic and EM observations up to time step $n$. One can compute the posterior PDF of $p\left(\kappa, \phi \mid \mathbf{d}_{o b s, 1: n}^{S e i s+E M}\right)$ by

$$
\begin{aligned}
p\left(\kappa, \phi \mid \mathbf{d}_{o b s, 1: n}^{S e i s+E M}\right) & =\int p\left(\kappa \mid \phi, S_{1: n}\right) p\left(\phi, S_{1: n} \mid \mathbf{d}_{o b s, 1: n}^{S e i s+E M}\right) \mathrm{d} S_{1: n}, \\
& \propto \int p\left(\kappa \mid \phi, S_{n}\right) p\left(\mathbf{d}_{o b s, n}^{\text {Seis }+E M} \mid \phi, S_{n}\right) p\left(\phi, S_{n} \mid \mathbf{d}_{o b s, 1: n-1}^{\text {Seis }+E M}\right) \mathrm{d} S_{n},
\end{aligned}
$$


where

$$
p\left(\phi, S_{n} \mid \mathbf{d}_{o b s, 1: n-1}^{S e i s+E M}\right)=\int p\left(S_{n} \mid \kappa, \phi\right) p\left(\kappa, \phi \mid \mathbf{d}_{o b s, 1: n-1}^{S e i s+E M}\right) \mathrm{d} \kappa .
$$

The formula 11 indicates a simultaneous assimilation scheme in which all data are integrated at the same time via the two-step integration procedure as described previously. In contrast, the formula 12 is derived by assuming that the data collected at different times are independent and the data at a particular time depend only on the state at this time, showing a recursive assimilation scheme in which the data are integrated sequentially in time as they become available. In this paper, we focus on the sequential assimilation scheme as dictated by the formula 12 .

Proper sampling of the initial ensemble characterizing the prior uncertainty is essential to ensure the performance of ensemble-based data-assimilation methods, in the sense that the posterior solution is constrained within the subspace of the initial ensemble of model parameters $[1,42]$. Under the ensemble formulation, the formula 13 indicates that we can generate the prior realizations of $S_{n}$ by running the reservoir flow simulator using the updated ensemble of porosity and permeability at previous time (or with the initial ensemble at the starting time). Note that the forecast of $S_{n}$ can be initiated with $S_{n-1}$ and the updated porosity and permeability at time $t_{n-1}$ without the simulation rerun from time $t_{0}$, because the consistency of these model variables is retained (at Step 2) after each assimilation time.

\subsection{Iterative updating of model variables}

For weakly nonlinear and approximately Gaussian problems, the standard EnKF or ES usually works well. However, iterative versions of EnKF, or ES, would be necessary if the nonlinearity is strong. Here, we choose to use an iterative ensemble smoother named LM-EnRML [8], although there are a number of suitable iterative algorithms based on ES available $[13,24,34]$. In the approximate form of LM-EnRML, the update to the $i$ th realization of model parameters $\mathbf{m}_{i}^{\ell} \in \Re^{N_{m}}$ at the $\ell$ th iteration is written as,

$$
\delta \mathbf{m}_{i}^{\ell}=\Delta \mathbf{M}^{\ell} \Delta \mathbf{D}^{\ell \mathrm{T}}\left[\left(1+\lambda^{\ell}\right) \mathbf{I}_{N_{d}}+\Delta \mathbf{D}^{\ell} \Delta \mathbf{D}^{\ell \mathrm{T}}\right]^{-1} \mathbf{C}_{D}^{-1 / 2}\left(\mathbf{d}_{o b s, i}-\mathbf{g}\left(\mathbf{m}_{i}^{\ell}\right)\right),
$$


where $\mathbf{I}_{N_{d}} \in \Re^{N_{d} \times N_{d}}$ is an identity matrix; $\mathbf{C}_{D}$ is the covariance matrix of data noise, which is assumed to be diagonal in the examples considered in this paper; $\mathbf{d}_{o b s, i} \in \Re^{N_{d}}$ is a vector of perturbed data sampled from a multivariate Gaussian distribution $\mathcal{N}\left(\mathbf{d}_{o b s}, \mathbf{C}_{D}\right)$ and $\mathbf{d}_{o b s} \in \Re^{N_{d}}$ is the vector of observed data (see Eqs. B.3 and B.5); $\mathbf{g}(\cdot)$ denotes the operator mapping the model parameters to predicted data; and $\lambda^{\ell}$ is the LevenbergMarquardt (LM) tuning parameter at the $\ell$ th iteration. The matrices $\Delta \mathbf{D}^{\ell} \in \Re^{N_{d} \times N_{e}}$ and $\Delta \mathbf{M}^{\ell} \in \Re^{N_{m} \times N_{e}}$ are the normalized deviation ensemble of simulated data and the deviation ensemble of model parameters, respectively, denoted as

$$
\begin{aligned}
\Delta \mathbf{D}^{\ell} & =\frac{1}{\sqrt{N_{e}-1}} \mathbf{C}_{D}^{-1 / 2} \mathbf{D}^{\ell}\left(\mathbf{I}_{N_{e}}-\overline{\mathbf{1}}_{N_{e}}\right), \\
\Delta \mathbf{M}^{\ell} & =\frac{1}{\sqrt{N_{e}-1}} \mathbf{M}^{\ell}\left(\mathbf{I}_{N_{e}}-\overline{\mathbf{1}}_{N_{e}}\right),
\end{aligned}
$$

where $\mathbf{I}_{N_{e}} \in \Re^{N_{e} \times N_{e}}$ is an identity matrix; $\overline{\mathbf{1}}_{N_{e}} \in \Re^{N_{e} \times N_{e}}$ is a matrix with all elements equal to $1 / N_{e}$; and $N_{e}$ is the ensemble size. Each column in $\mathbf{M}^{\ell} \in \Re^{N_{m} \times N_{e}}$ and $\mathbf{D}^{\ell} \in$ $\Re^{N_{d} \times N_{e}}$ respectively represents one realization of model parameters $\mathbf{m}_{i}^{\ell}$ and corresponding simulated data $\mathbf{g}\left(\mathbf{m}_{i}^{\ell}\right)$.

It has been well recognized that two problems become prominent in a standard implementation of an ensemble method with a limited ensemble size [1]: (i) spurious correlations arising in the ensemble approximation of the covariance matrix impose changes to model or state variables in regions that should not be updated, (ii) limited number of degrees of freedom constrains the ability to assimilate large amounts of independent data. In the case of crosswell seismic and EM measurements, the number of data can become very large, and the redundancy of the measurements is potentially high due to the dense and repeated spatial coverage of the interwell region. Therefore, it necessitates the use of some regularization to overcome the mentioned issues.

In [60], a distance-based Kalman gain localization was applied to integrate crosswell EM data, which was shown to be a viable option if the localization matrix was determined appropriately. However, choosing a proper localization matrix is not an easy task. It usually involves a joint consideration of prior ensemble covariance, data sensitivity and ensemble size. This becomes particularly difficult for the full-waveform seismic data con- 
sidered in this paper, due to their complex sensitivity patterns with respect to related model variables. To circumvent these difficulties, we adopt a non-distance-based Kalman gain localization method introduced by Zhang and Oliver [58]. It applies the bootstrap sampling to assess the confidence level of each element in the Kalman gain matrix, so that the real and the spurious correlations in the Kalman gain matrix can be discriminated. The localized Kalman gain is given by

$$
\mathbf{K}^{l o c}=\mathbf{L} \circ \underbrace{\left\{\Delta \mathbf{M}^{\ell} \Delta \mathbf{D}^{\ell \mathrm{T}}\left[\left(1+\lambda^{\ell}\right) \mathbf{I}_{N_{d}}+\Delta \mathbf{D}^{\ell} \Delta \mathbf{D}^{\ell \mathrm{T}}\right]^{-1}\right\}}_{\text {Kalman gain } \mathbf{K}},
$$

where $\mathbf{L}$ is the localization matrix and o denotes the Schur product or element-wise multiplication. The element $\mathbf{L}_{i j}$ named confidence factor in the localization matrix is given by

$$
\begin{aligned}
\mathbf{L}_{i j} & =\frac{1}{1+\mathbf{R}_{i j}^{2}\left(1+1 /\left(\gamma^{2}\right)\right)}, \\
\mathbf{R}_{i j}^{2} & =\frac{\frac{1}{N_{b}} \sum_{l=1}^{N_{b}}\left(\mathbf{K}_{i j}^{l}-\mathbf{K}_{\mathbf{i j}}\right)^{2}}{\mathbf{K}_{i j}^{2}}, \text { for } i=1,2, \cdots, N_{m}, j=1,2, \cdots, N_{d},
\end{aligned}
$$

where $\mathbf{K}_{i j}^{l}$ represents the element from the $l^{\text {th }}$ bootstrapped sample of the Kalman gain matrix and $N_{b}$ is the number of bootstrapped samples. The weighting factor $\gamma$ is introduced to regulate the estimate of $\mathbf{L}$ to avoid taking negative values and adjust the degree of screening to spurious correlations. The value of $\mathbf{L}_{i j}$ reflects the accuracy of the correlations present in the Kalman gain matrix. Small value of $\mathbf{L}_{i j}$ indicates an unreliable correlation of the corresponding model variable with the data, which thus should be eliminated or suppressed. By decreasing the value of $\gamma$, the confidence factor $\mathbf{L}_{i j}$ will get close to 0 especially for the ones with large variance, so that the Kalman gain is increasingly screened. Because $\mathbf{L}_{i j}$ is never equal to 0, the suppressed spurious correlations will be gradually added back to the model update with iteration. It thus allows for the selection of a lower value of $\gamma$ compared with the case without iteration. On the other hand, reducing the value of $\gamma$ will slow down the convergence rate so that more iterations are required. In our experiments, the value of $\gamma$ is determined by experimentation. 


\subsection{Distance parameterization of saturation fronts}

A distance parameterization method $[29,57]$ is employed to facilitate the assimilation of the inverted saturation field from seismic and EM data. The distance parameterization utilizes the shape information of the extracted features from the attribute of interest. Let $A$ and $B$ represent the water fronts (i.e., contours) extracted from the inverted and the simulated saturation fields, respectively. The corresponding binary images (e.g., with 0 indicating the background and with 1 identifying the shape) are denoted by the matrices $I_{A}$ and $I_{B}$. Three parameterization schemes were developed in Zhang and Leeuwenburgh [57]. Here, we choose the scheme termed as LHDC (local Hausdorff distance based on contours),

$$
\operatorname{LHDC}(A, B)=I_{A} \circ D_{B}+I_{B} \circ D_{A},
$$

where $D_{A}$ and $D_{B}$ are two distance maps for contours $A$ and $B$, respectively. The distance maps can be computed efficiently using a fast marching method [56]. The value at each point of a distance map measures the distance from this point to the nearest point on a contour. Additionally, signed distance fields are used such that distance information discriminates the points inside and outside of a contour. Positive distance values outside of the contour and negative distance values inside the contour are assigned. In essence, LHDC quantifies the differences with two directed distance maps in complementary directions, $I_{A} \circ D_{B}$ (distance from $B$ to $A$ ) and $I_{B} \circ D_{A}$ (distance from $A$ to $B$ ).

Fig. 3 shows a stepwise diagram illustrating the computation of the LHDC as a measure of local dissimilarity for two saturation fronts. After applying LHDC, the interpreted front (Shape A) corresponds to zero distance map (i.e., $\left.\mathbf{d}_{o b s}=\operatorname{LHDC}(A, A)=0\right)$ and the

simulated front (Shape B) corresponds to the dissimilarity distance map (i.e., $\mathbf{d}_{s i m, j}=$ $\left.\operatorname{LHDC}\left(A, B_{j}\right)\right)$, which are subsequently integrated by the LM-EnRML as in Eqs. (14) and (17). It is conceivable that $\mathbf{d}_{s i m}$ will decrease to zero distance map as the simulated fronts get closer to the interpreted fronts during the history matching process. 


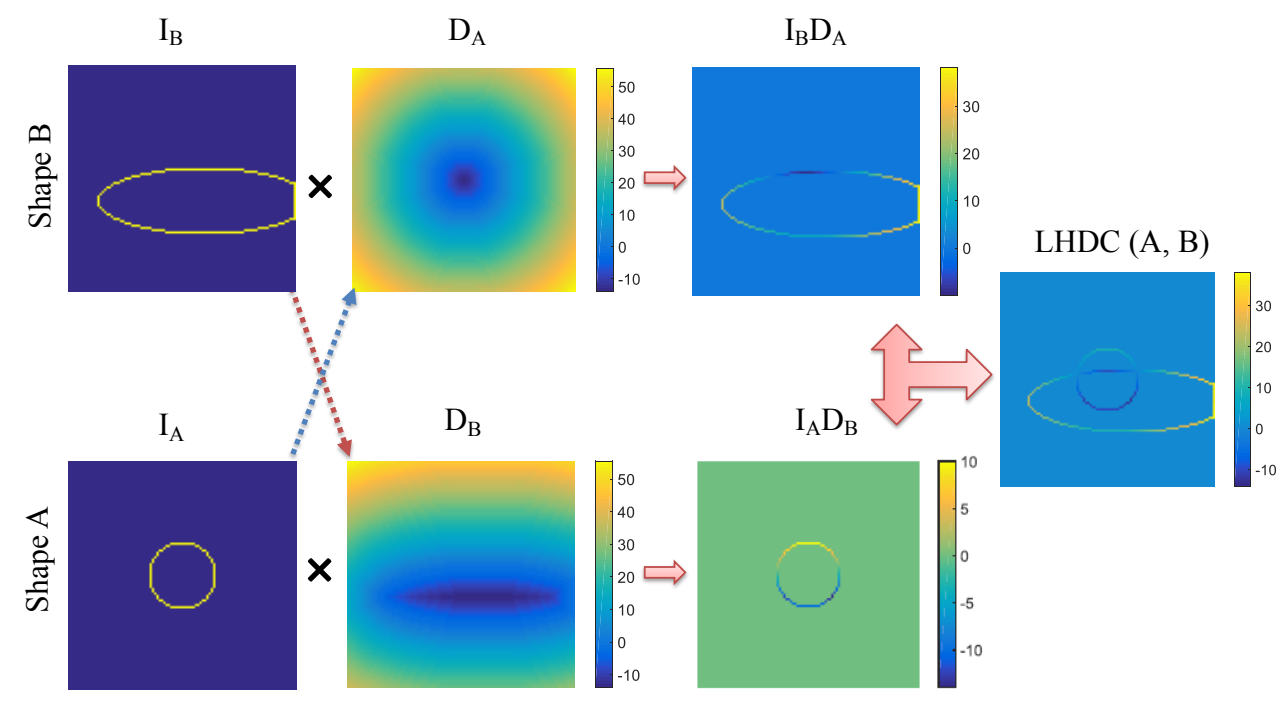

Fig. 3: Schematic diagram of LHDC as in Eq. (20) for shape comparison based on contours.

\section{Application}

We examine the performance of the developed history-matching workflow using a two-dimensional (2D) channelized reservoir model and a realistic three-dimensional (3D) reservoir model with a crosswell configuration for EM and seismic surveys.

\subsection{Channelized 2D reservoir model}

Model setup. The reference porosity and log-transformed permeability fields (used to generate pseudo-observations) are shown in Fig. 4 displaying channel-like features oriented in the east-west direction. The reservoir model has the dimensions of $45 \times 45 \times 1$ with a uniform grid size of $5 \mathrm{~m}$ in the $x$ - and $y$-directions, and $20 \mathrm{~m}$ in the $z$-direction. There are two facies types in the reference model. Realizations of facies model are first generated with a multipoint-based geostatistical algorithm [52]. Conditioned on the facies models, realizations of porosity and log-permeability are then generated using the sequential Gaussian simulation algorithm with an exponential variogram model with an isotropic range of 8 gridblocks. The reference model is randomly drawn from the generated realizations. As shown in Fig. 4, the channel facies (sand) is characterized by high permeability and porosity while the background facies (shale) exhibits opposite features.

The fluid system consists of two immiscible phases (oil and brine) with a connate 
water saturation of 0.2 , residual oil saturation of 0.2 , and initial formation pressure of 310 bar. There are two horizontal wells, one producer (shown as solid black circles) and one injector (shown as white crosses), with an interwell separation of $125 \mathrm{~m}$. The producer is under bottom-hole pressure (BHP) control at 138 bar, and the injector is on rate control at $200 \mathrm{sm}^{3} /$ day. The input parameters of the rock-physics models used in this experiment are summarized in Table A.1 of Appendix A.

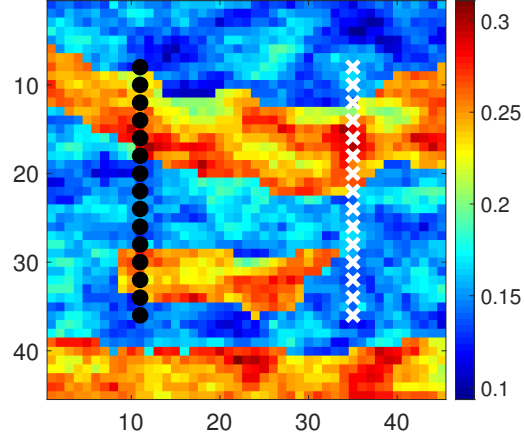

(a) Reference porosity

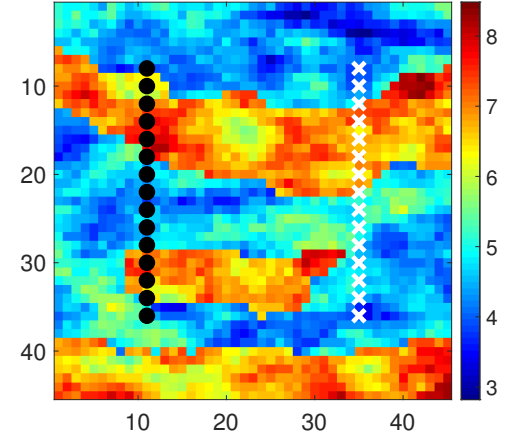

(b) Reference log-permeability

Fig. 4: The reference porosity and log-permeability fields from which synthetic historical data are generated. Trajectories of the horizontal injector are shown as white crosses and the horizontal producer are shown as solid black circles.

Measurement setup. For the crosswell configuration of both seismic and EM surveys, transmitters and receivers are placed in the boreholes of the producer and the injector as shown in Fig. 5, respectively. There are 15 transmitters and 15 receivers that are uniformly distributed from the heel to the toe of each horizontal well. In the crosswell EM surveys, the transmitters are axial magnetic dipoles, and the measured data are the horizontal components of the magnetic fields. The frequency of operation is $500 \mathrm{~Hz}$. In the crosswell seismic surveys, monopole sources are used, and the receivers measure scalar pressure fields. We choose to use the data that correspond to operating frequencies of 20, 60 and $100 \mathrm{~Hz}$ empirically for the inversion. The EM model has the dimensions of $64 \times 64 \times 16$, with a uniform grid size of $5 \mathrm{~m}$ within the reservoir, which is the domain of interest. It has the same lateral resolution as the reservoir flow model. Away from the domain of interest, a variable spacing is chosen for computational efficiency so that 
perfectly conducting boundary conditions can be satisfied. The seismic model has the dimensions of $45 \times 45 \times 7$ with the same grid size as the EM model. All three model grids are superposed within the domain of interest. Time-lapse crosswell seismic and EM surveys are conducted at days 60 and 180, respectively. The reference saturation fields at these survey times are shown in Fig. 6. Note that the measurement setup considered here may be somewhat simplified, in the sense that the acquisition and design of real seismic and EM surveys usually involve many other technical factors and practical considerations [55]. The synthetic seismic and EM data are corrupted with 5\% Gaussian random white noise. The total number of seismic and EM data for each survey amounts to 1800 (= $15 \times 15 \times 2 \times 3^{\text {Seis }}+15 \times 15 \times 2^{E M}$, see Appendix B for more detail).

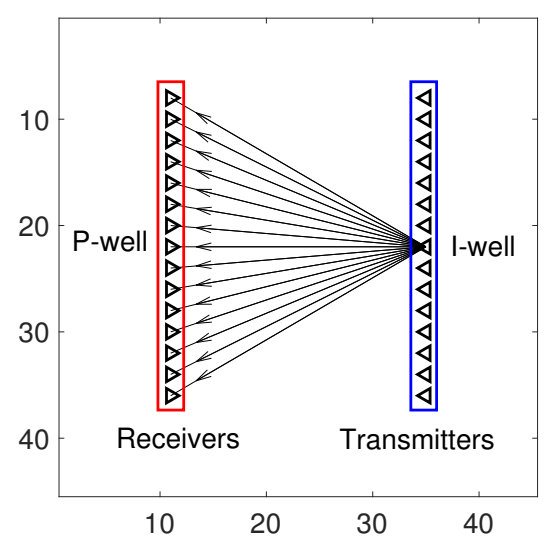

Fig. 5: Crosswell seismic and EM survey configuration.

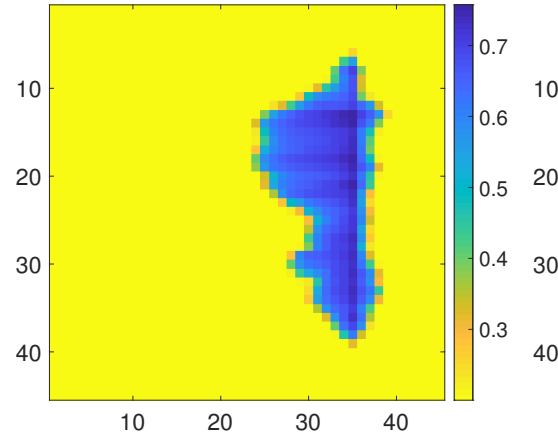

(a) Day 60

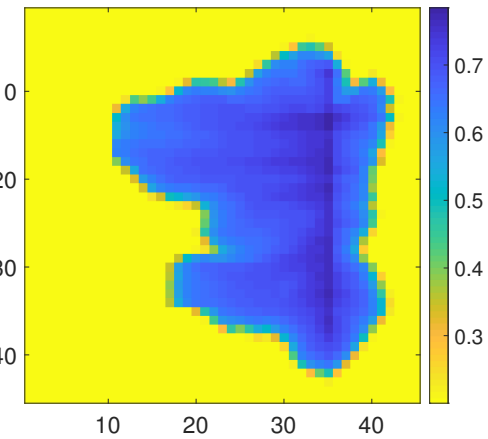

(b) Day 180

Fig. 6: The reference saturation fields for the crosswell seismic and EM surveys conducted at days 60 and 180, respectively. 
History-matching setup. The initial ensemble consists of 100 realizations for each type of model variables, which are not conditioned on well data. For the LM-EnRML, the starting value of the LM tuning parameter $\lambda$ is chosen empirically of the same order of

magnitude as $\sum_{j=1}^{N_{e}} S_{d}\left(\mathbf{m}_{j}^{0}\right) /\left(2 N_{e}\right)$, where $m^{0}$ is the initial model variables and the data mismatch $S_{d}$ is defined by

$$
S_{d}\left(\mathbf{m}_{j}\right)=\left[\mathbf{d}_{o b s}-\mathbf{g}\left(\mathbf{m}_{j}\right)\right]^{\mathrm{T}} \mathbf{C}_{D}^{-1}\left[\mathbf{d}_{o b s}-\mathbf{g}\left(\mathbf{m}_{j}\right)\right]
$$

We follow the tuning strategy used in Chen and Oliver [8]. At each iteration, if both the ensemble mean and the standard deviation of $\left\{S_{d}\left(\mathbf{m}_{j}\right)\right\}_{j=1}^{N_{e}}$ are reduced, the model update is accepted and the value of $\lambda$ is divided by 10; if only the mean of the data mismatch is reduced, the model update is accepted but the value of $\lambda$ is kept unchanged; otherwise, if the mean of the data mismatch is increased, the model update is rejected, and the value of $\lambda$ is multiplied by 10. Regarding the stopping criteria, the iterative updating stops when the number of iterations exceeds 15 , or the reduction of the mean of $\left\{S_{d}\left(\mathbf{m}_{j}\right)\right\}_{j=1}^{N_{e}}$ after two consecutive iterations is less than $1 \%$. The weighting factor $\gamma=0.3$ is used for the bootstrap-based Kalman gain localization, which implies a relatively strong localization. The value of $\gamma$ can vary between different types of model variables, but for simplicity, the same $\gamma$ value is applied here unless specified explicitly.

To analyze the synergy effect by the joint use of seismic and EM data, we conduct three experiments in which the first one history matches only EM data, the second one history matches only seismic data, and the last one history matches both types of data. The time-lapse seismic and EM data are assimilated sequentially in time in all cases.

\subsubsection{Case I: Neglect porosity-permeability dependence}

In this comparative experimentation, we ignore the dependence between porosity and permeability distributions, so that the permeability distribution is conditioned only on the updated saturation distribution at the second integration step.

Day 60 - step 1. Fig. 7 shows the distribution of the data mismatch with iteration for separate and joint assimilation of seismic and EM data at day 60. The initial data mismatches for EM and seismic data are of similar orders of magnitude. In all cases, the 
data mismatch is reduced consistently along with the iterations and reaches an acceptable level.

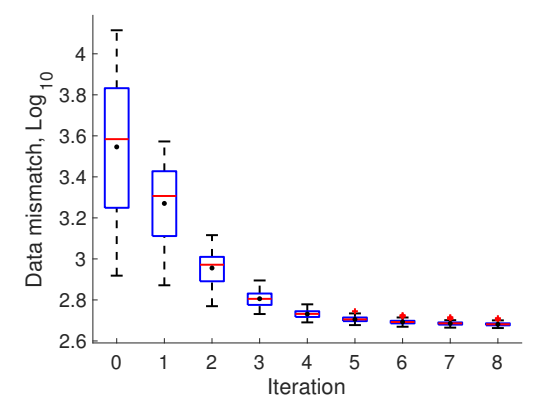

(a) EM only

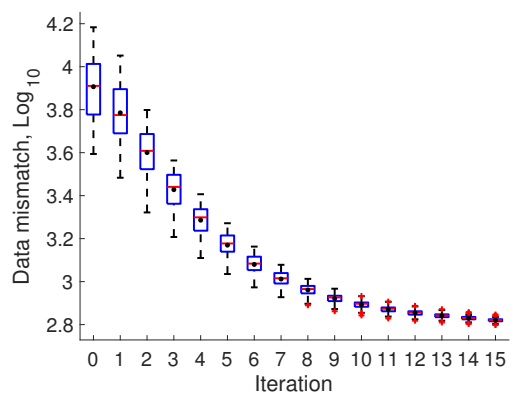

(b) Seismic only

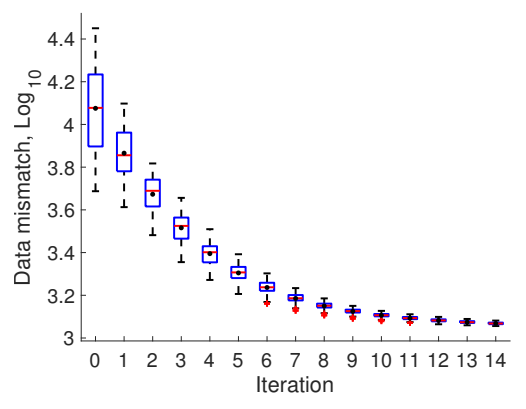

(c) Both

Fig. 7: Boxplots of data mismatch as functions of iteration step for separate and joint assimilation of seismic and EM data at day 60 . The bounds of the box are $25 \%$ and $75 \%$ quantiles, the whiskers are the extremes, the line in the box is the median, the dot is mean, and the pluses are outliers.

Fig. 8 compares the root-mean-square error (RMSE) of the updated ensembles of porosity and water saturation from the three experiments, in which the RMSE is defined as

$$
\mathrm{RMSE}=\sqrt{\frac{\sum_{j=1}^{N_{m}}\left(\mathbf{m}_{i, j}-\mathbf{m}_{t, j}\right)^{2}}{N_{m}}},
$$

where the subscripts $i, j$, and $t$ represent realization number, element index, and reference model property, respectively. The behavior of the change of RMSE along with the iterations somehow reflects the characteristics of the information content of each data type. For the EM only case, the reduction of RMSE for the saturation ensemble is larger and more consistent than the one for the porosity ensemble. By contrast, for the seismic only case, the reduction of RMSE for the porosity ensemble is stronger and more stable than the one for the saturation ensemble. The complementary effect is seen when both types of data are assimilated. The seismic data plays a dominant role in the model update of this specific example, partly because EM data are collected in single frequency while seismic data contain multiple-frequency components.

Fig. 9 shows the means and standard deviations of the porosity field for the initial ensemble and the updated ensembles for the three cases. The initial mean exhibits a rather 


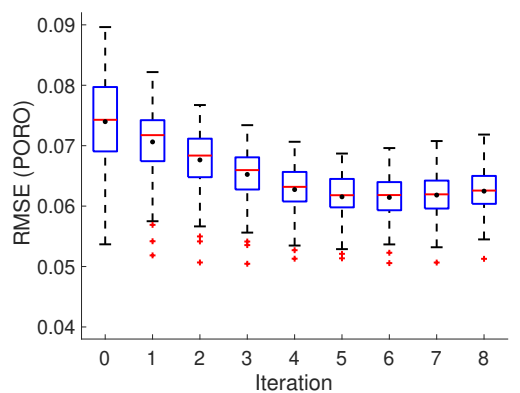

(a) EM only - porosity

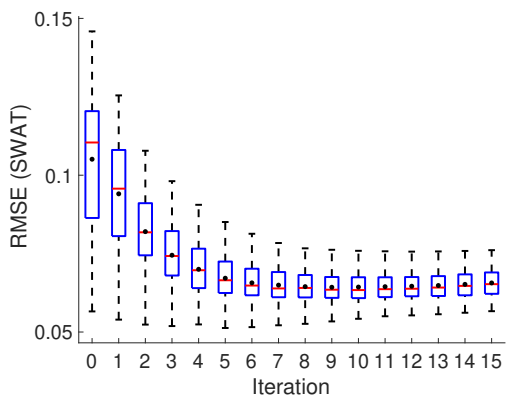

(d) Seismic only - saturation

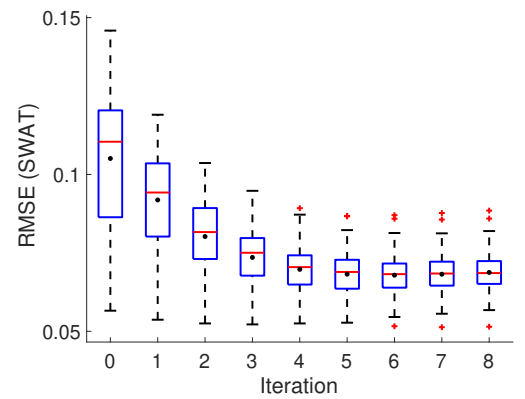

(b) EM only - saturation

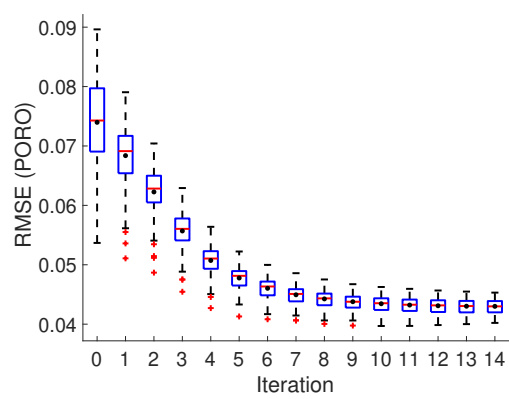

(e) Both - porosity

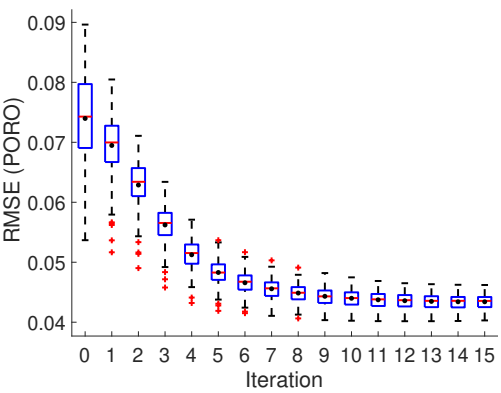

(c) Seismic only - porosity

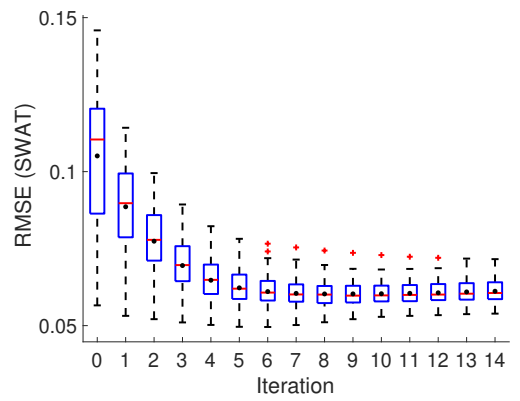

(f) Both - saturation

Fig. 8: Boxplots of RMSEs of porosity and water saturation as functions of iteration step for separate and joint assimilation of seismic and EM data at day 60 . 
homogeneous profile with large standard deviation over the entire field. When only EM data are integrated, some channel features observed in the reference porosity field appear in the updated mean field. Reduced variability concentrates in the neighborhood of well locations, particularly around the injector. For the other cases in which seismic data are incorporated, a significantly refined channel profile in better agreement with the reference field is observed. Meanwhile, the standard deviation fields are further reduced prominently across the whole domain, particularly in the interwell region. In contrast, the difference in the mean fields of saturation for the three cases shown in Fig. 10 is less obvious, even though the difference in the standard deviation fields is still dramatic. In general, the mean saturation field in all cases reproduces the main features observed in the reference saturation field as shown in Fig. 6(a).

Day 60 - step 2. We proceed with the second step to condition the permeability field upon the inverted saturation field. Fig. 11 summaries the process of determination and extraction of saturation fronts from the updated mean saturation fields for the three cases. To reduce inversion artifacts and extract coherent features, we apply a lateral smoothing to the mean saturation fields as shown in the first column of Fig. 11. A threshold value is necessary in order to derive the interpreted front positions. Empirically, we determine the threshold value by first identifying the unstable or fast-changing areas in the contour map of the smoothed mean saturation field, as shown in the middle column of Fig. 11 highlighted by the black rectangle. In these areas, saturation fronts change dramatically so that biases are prone to be introduced when the threshold value is 'overly small', or important information can be lost when the threshold value is 'overly large'. It is therefore wise to take a moderate threshold value to avoid extremes. When the saturation fronts change relatively smoothly as in the EM only case (the top subfigure in the middle column of Fig. 11), it generally allows for a higher tolerance for the threshold value and is less prone to introduce bias from the selection. Taking these factors into account, we select a threshold value of 0.3 for all the three cases to derive the interpreted front positions (corresponding to the red contours in the middle column of Fig. 11). Uncorrelated distance measurement errors are assumed with a standard deviation of $5 \mathrm{~m}$ 


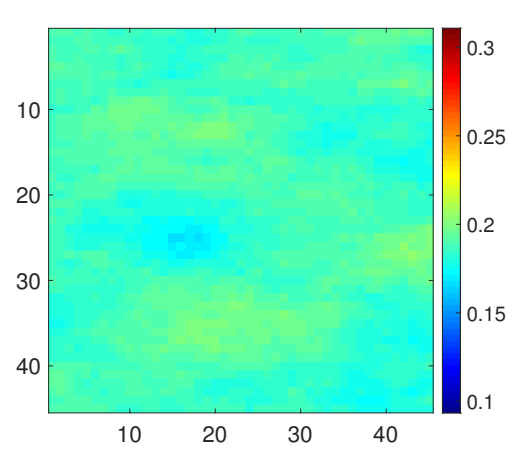

(a) Mean - initial

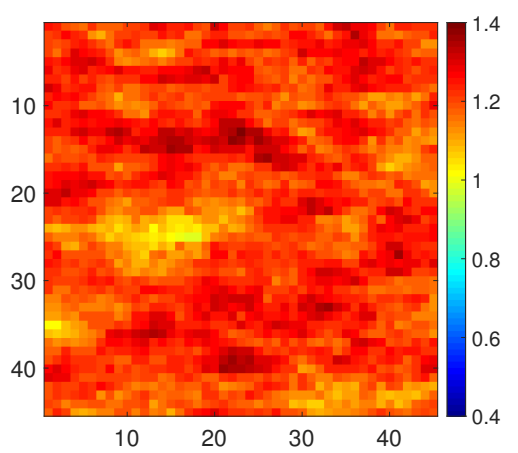

(b) STD - initial

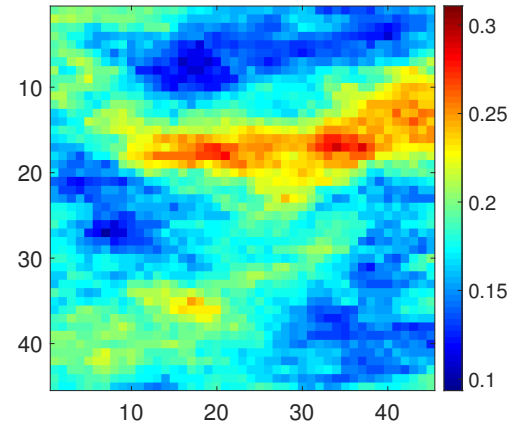

(c) Mean - EM only

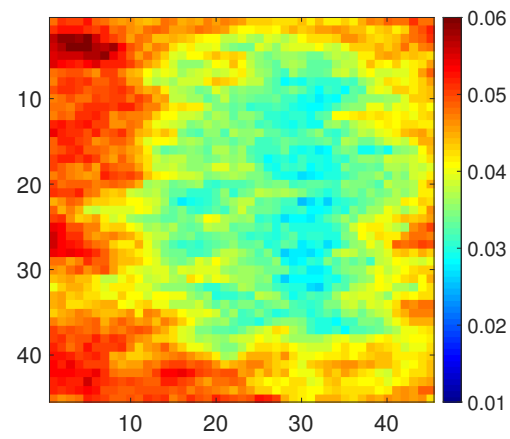

(f) STD - EM only

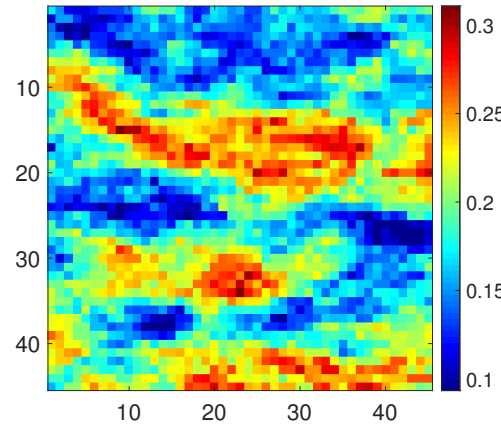

(d) Mean - seismic only

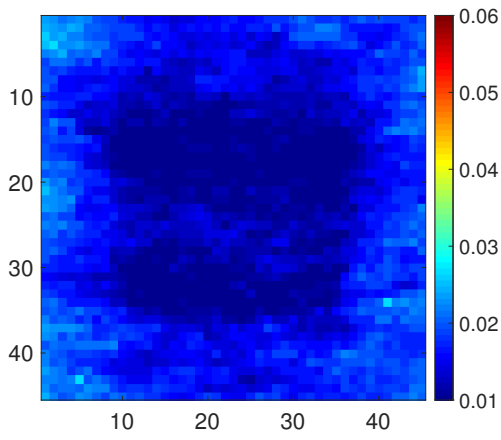

(g) STD - seismic only

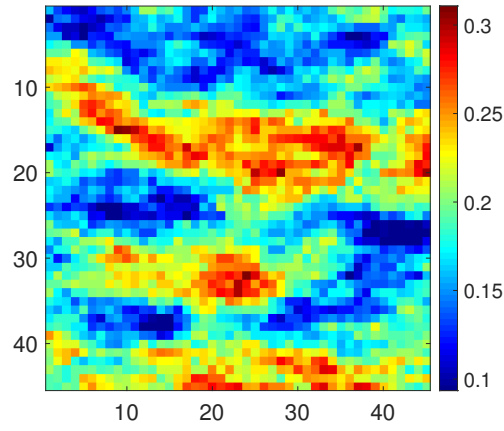

(e) Mean - both

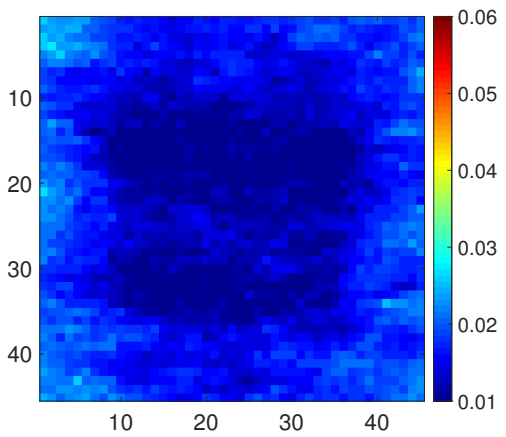

(h) STD - sboth

Fig. 9: The means and standard deviations (STD) of porosity for the initial ensemble ( $a$ and $b$ ) and the updated ensembles (c through $\mathrm{h}$ ) after separate and joint assimialtion of seismic and EM data at day 60 . 

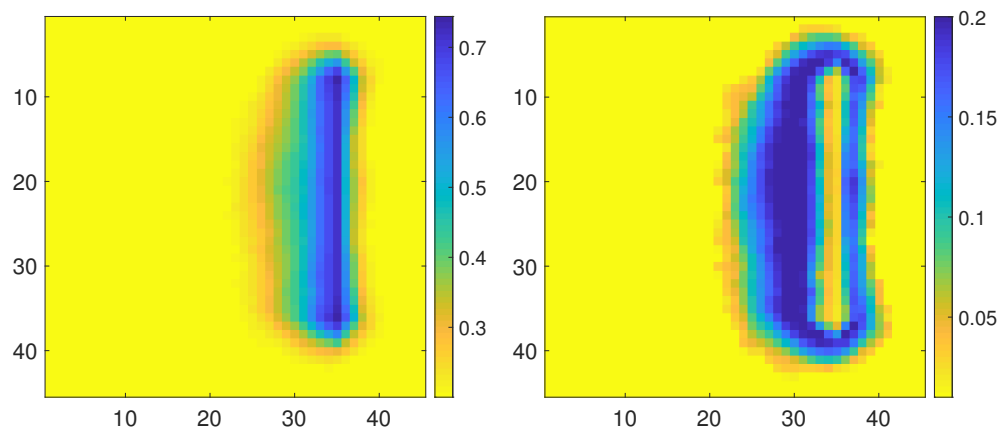

(a) Mean - initial

(b) STD - initial

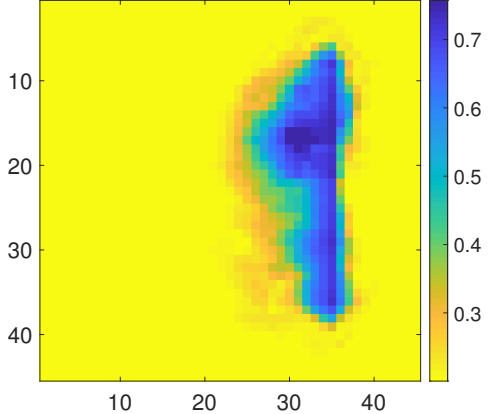

(c) Mean - EM only

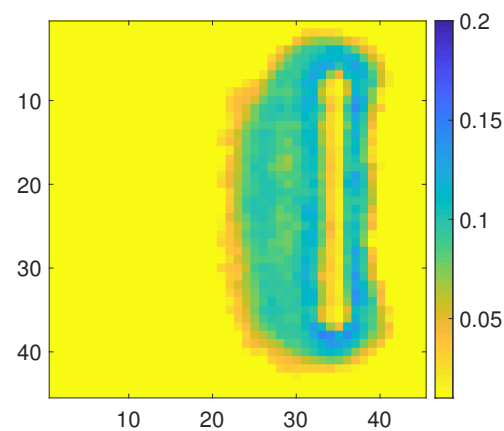

(f) STD - EM only

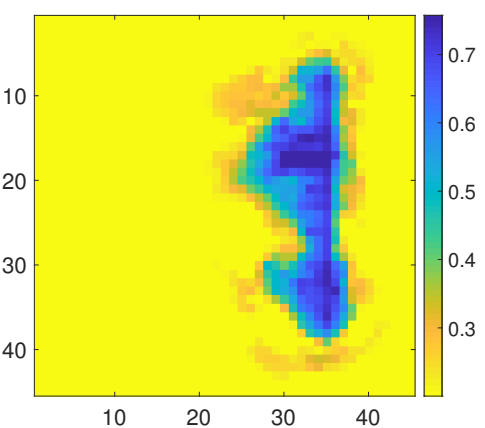

(d) Mean - seismic only

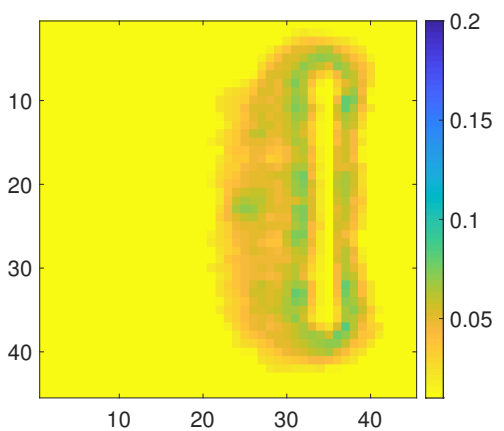

(g) STD - seismic only

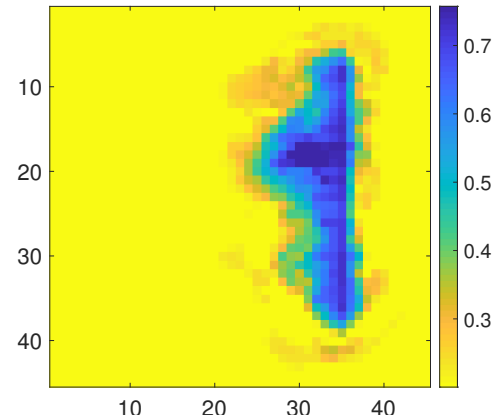

(e) Mean - both

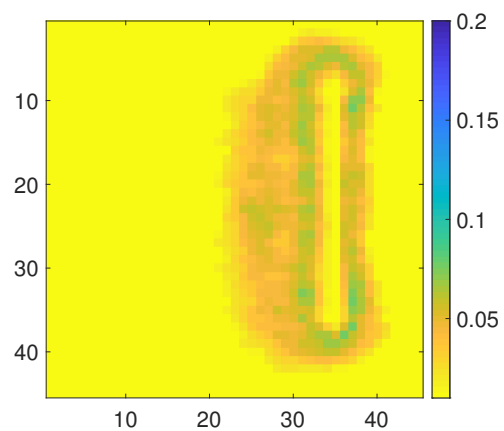

(h) STD - both

Fig. 10: The means and standard deviations of water saturation for the initial ensemble (a and b) and the updated ensembles (c through h) after separate and joint assimilation of seismic and EM data at day 60. 
(one grid cell length), which is roughly estimated according to the spread of the extracted fronts from the updated saturation ensembles as shown in the rightmost column of Fig. 11. Clearly, the interpreted front positions (in red dots) match the true front positions (in green circles) extracted from the reference saturation field (in Fig. 6(a)) for the most part in all cases. The joint incorporation of seismic and EM data shows a positive synergy effect on the characterization of saturation fronts, from which the best result is obtained.

The extracted saturation fronts are then assimilated to calibrate the initial permeability ensemble using the LM-EnRML with the distance parameterization. Fig. 12 shows a consistent reduction of both the data mismatch and the RMSE of log-permeability during the iteration with comparable final results for all cases. Fig. 13 displays the mean and standard deviation fields of the predicted saturation ensembles at day 60 after the second assimilation step. For each case, a good match to the corresponding interpreted front is obtained with reduced variation concentrating around the front locations.

Fig. 14 compares the means and standard deviations of the initial ensemble and the updated ensembles of log-permeability after the assimilation of saturation fronts at day 60. The updated ensemble means for all cases roughly reproduce the top high-permeable channel structure observed in Fig. 4(b). It reflects the essential information carried by the interpreted saturation fronts. The standard deviations exhibit similar spatial patterns for all cases with dramatic reduction in the surrounding area of the injector, from where the water fronts originate.

Day 180 - step 1. We proceed to the next survey time with the updated ensemble of porosity and permeability at the last time step. Fig. 15 shows the prior ensemble of water saturation at day 180 generated via forward flow simulation using the updated ensemble of porosity and permeability. Because of the drastic reduction in the variation of the saturation distribution at day 60 , the variability of the predicted saturation ensemble at day 180 decreases significantly as well. Fig. 16 shows the distribution of the data mismatch along with the iterations for separate and joint assimilation of seismic and EM data at day 180 . 

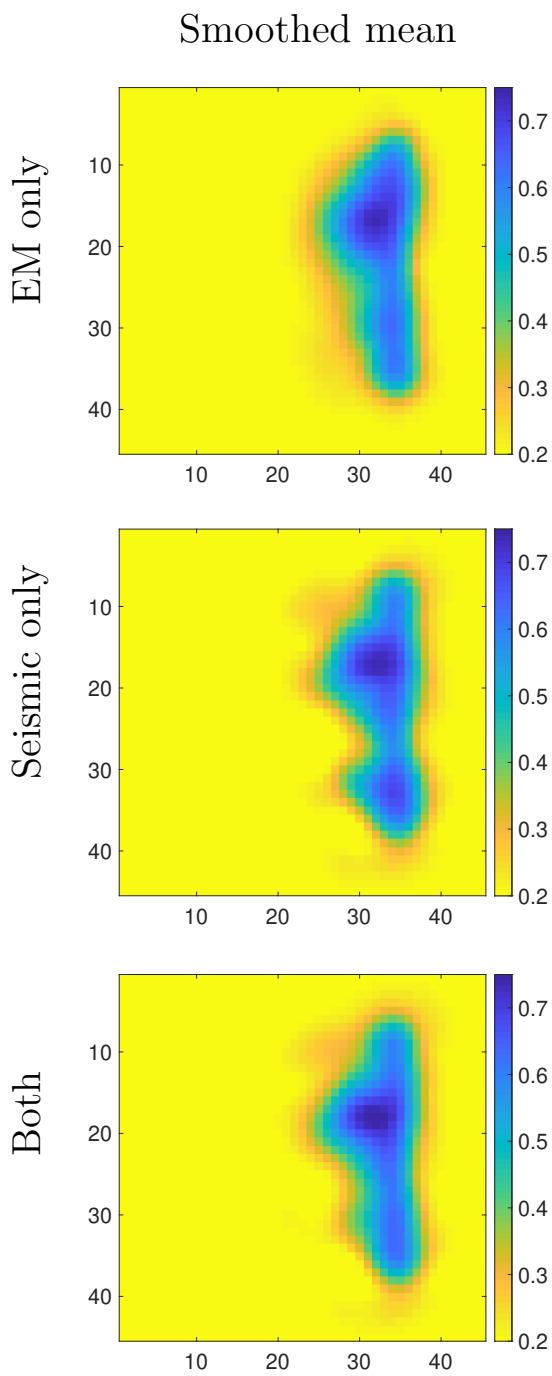

Truncated contours
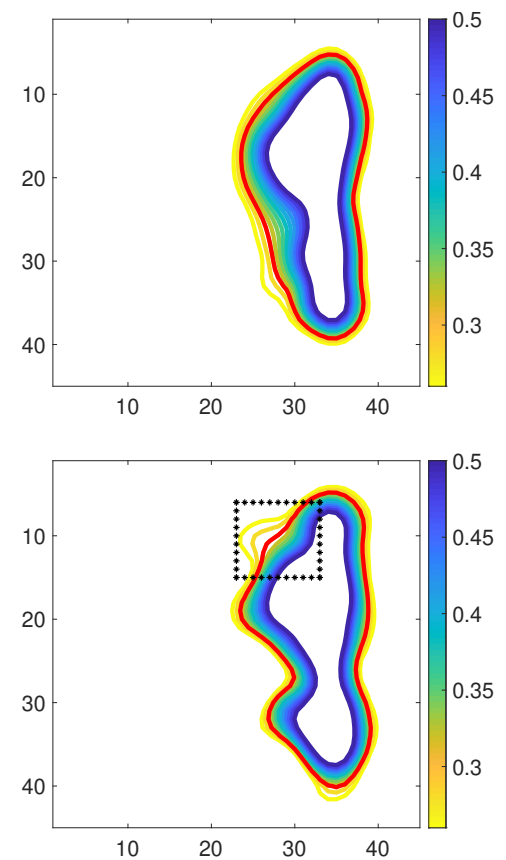

Extracted fronts
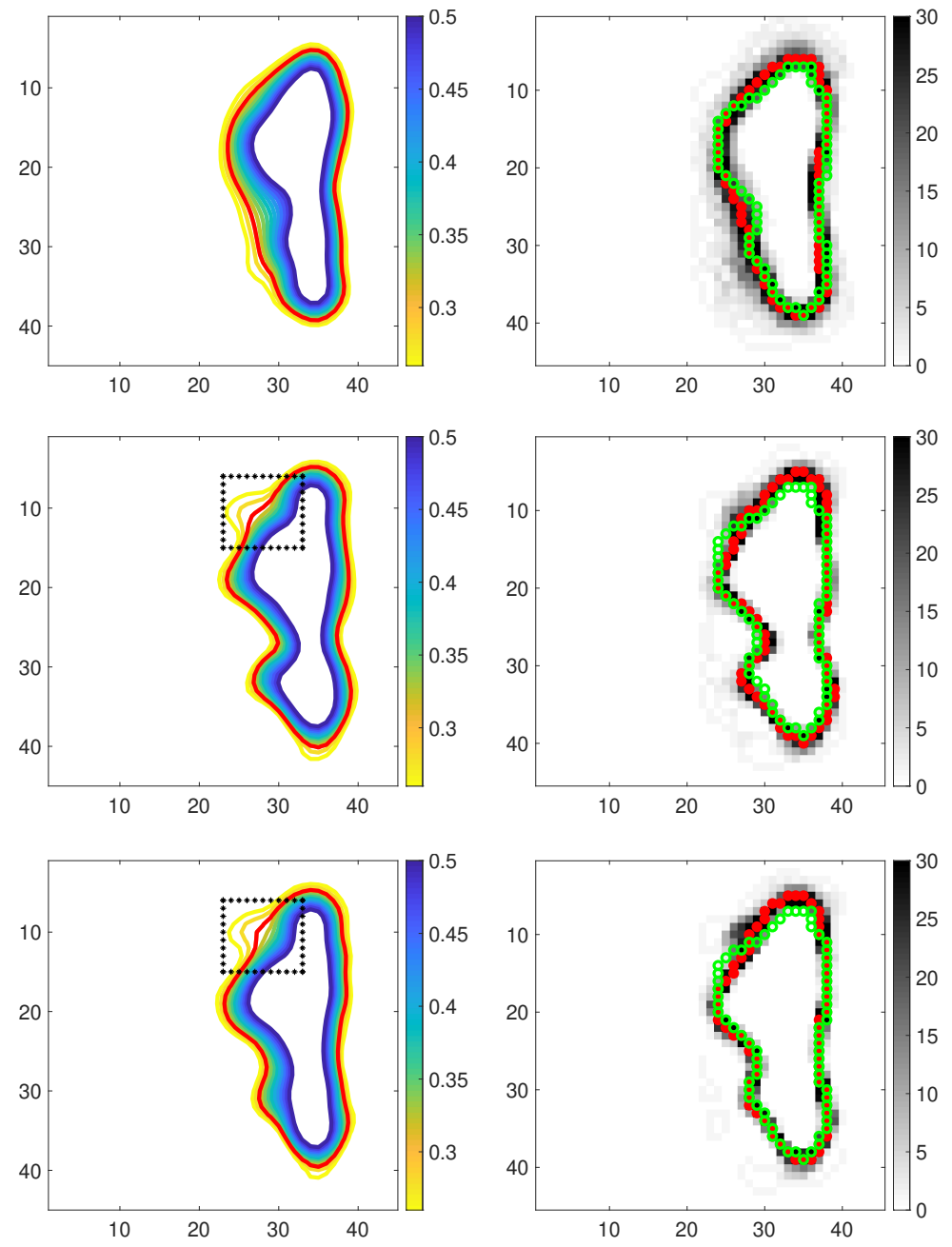

Fig. 11: Determination and extraction of saturation fronts from the inverted mean saturation fields at day 60. In the middle column, the black rectangle highlights the unstable changing area of saturation contours and the red isoline corresponding to 0.3 indicates the interpreted front selected for history matching. In the rightmost column, the true front positions extracted from the reference saturation field are marked with green circles. For comparison, the interpreted front locations are marked with red dots. The grayscale indicates the count of occurrence of water front at a location extracted from the updated ensemble of saturation fields. The upper colormap limit is mapped from 100 (the ensemble size) to 30 for better visibility. 


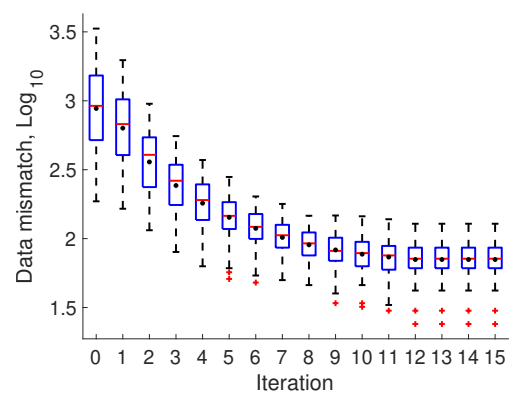

(a) EM only - data mismatch

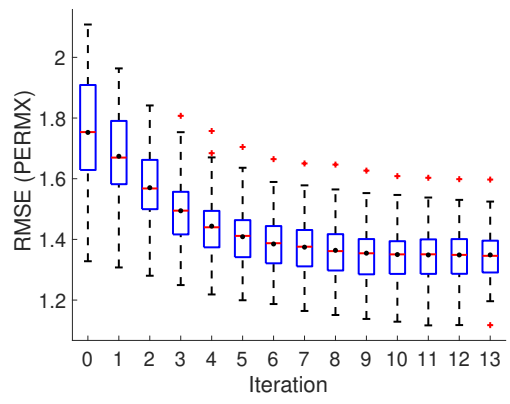

(d) Seismic only - RMSE

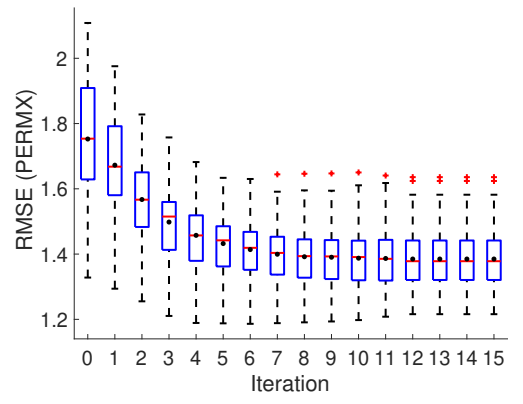

(b) EM only - RMSE

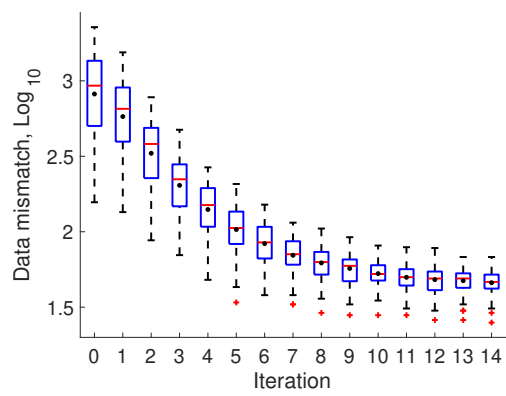

(e) Both - data mismatch

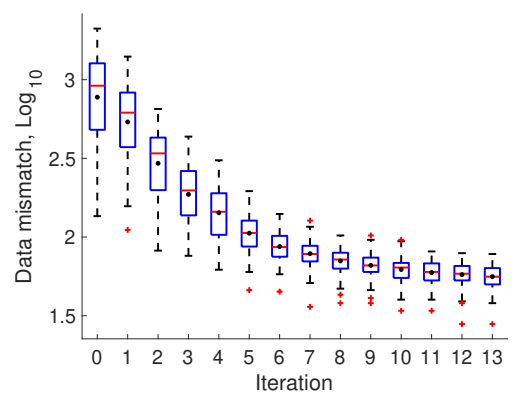

(c) Seismic only - data mismatch

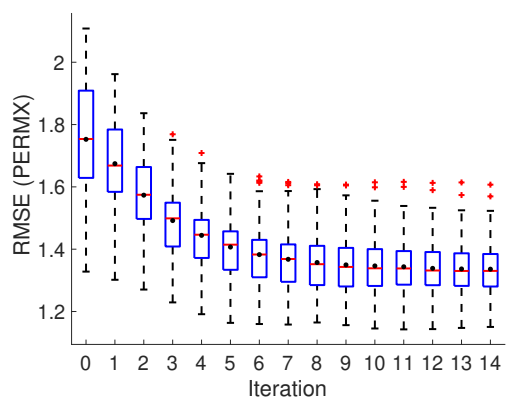

(f) Both - RMSE

Fig. 12: Boxplots of data mismatch and the RMSE of log-permeability as functions of iteration step after the assimilation of saturation fronts at day 60 . 


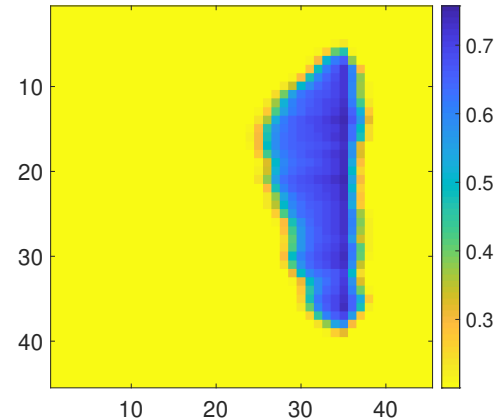

(a) Mean - EM only

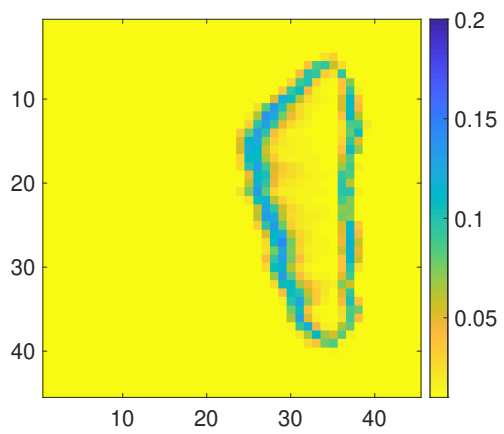

(d) STD - EM only

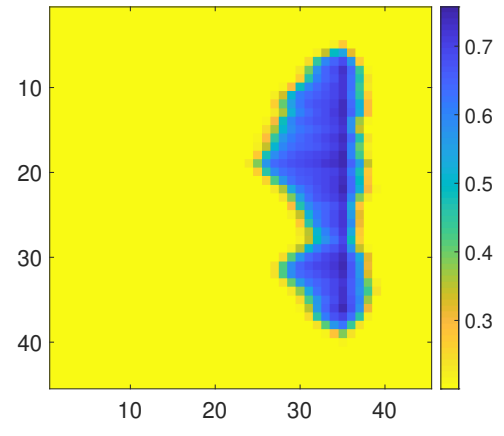

(b) Mean - seismic only

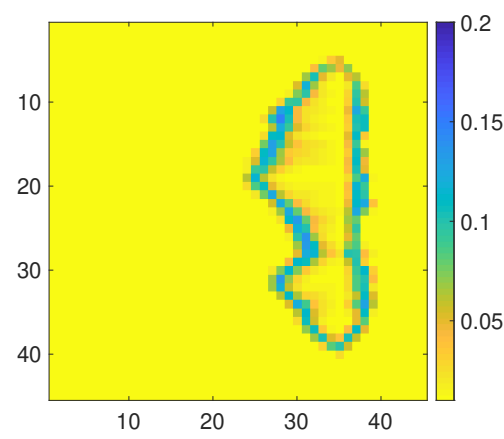

(e) STD - seismic only

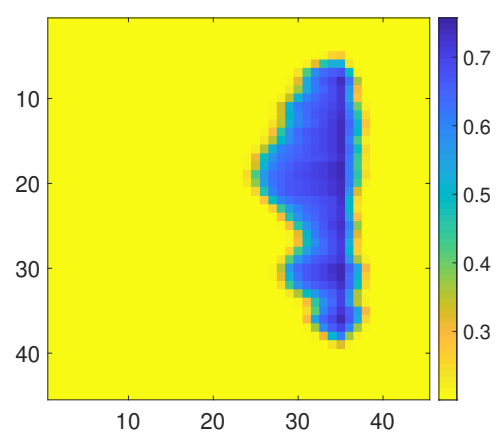

(c) Mean - both

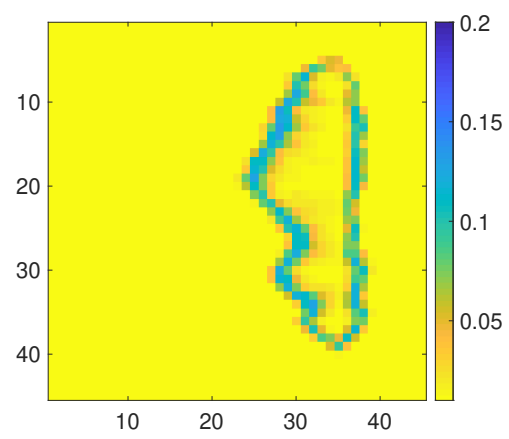

(f) STD - both

Fig. 13: The means and standard deviations of the predicted ensembles of water saturation at day 60 after the second assimilation step for the three cases. 


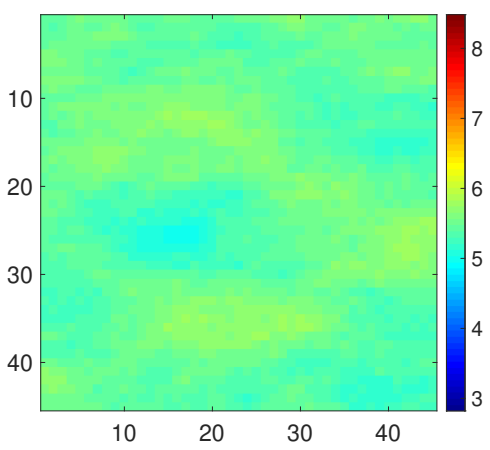

(a) Mean - initial

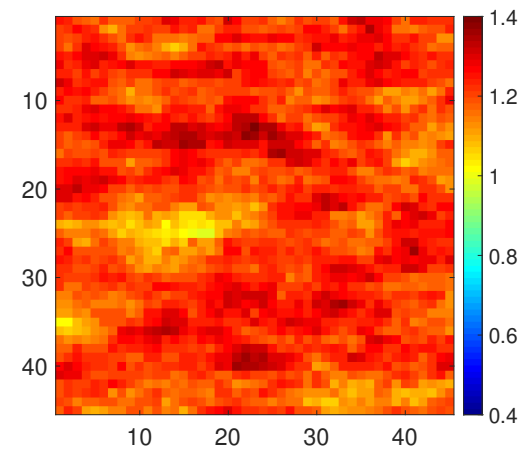

(b) STD - initial

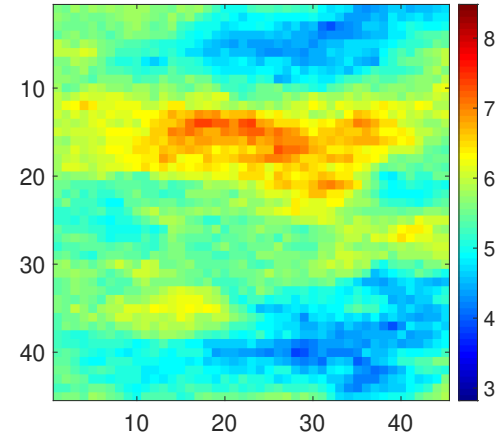

(c) Mean - EM only

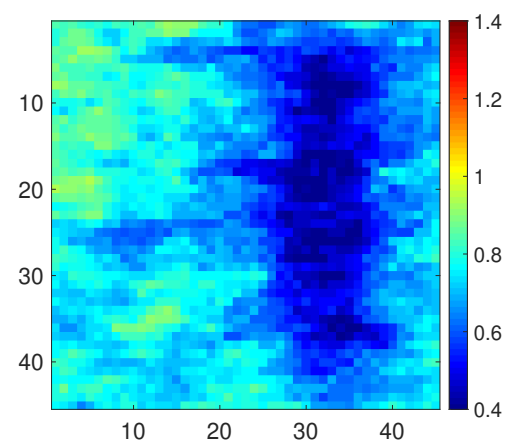

(f) STD - EM only

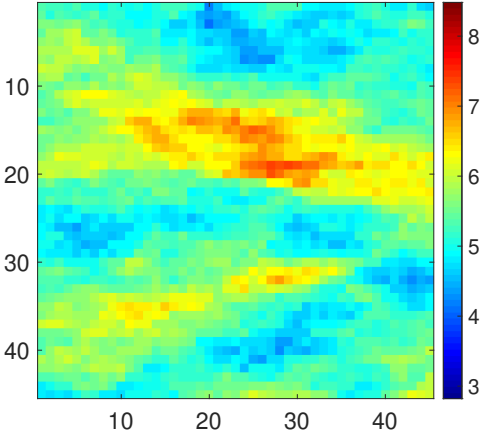

(d) Mean - seismic only

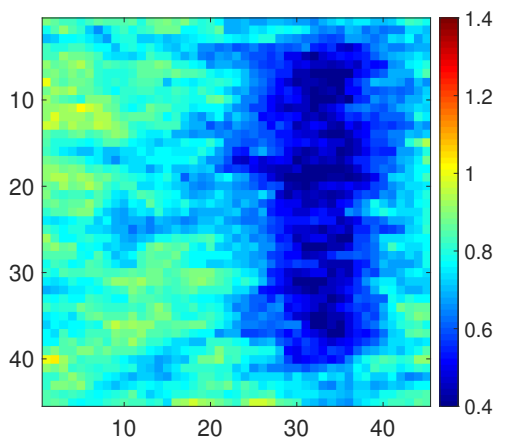

(g) STD - seismic only

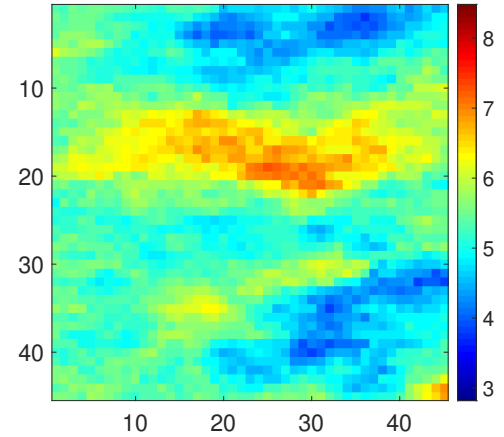

(e) Mean - both

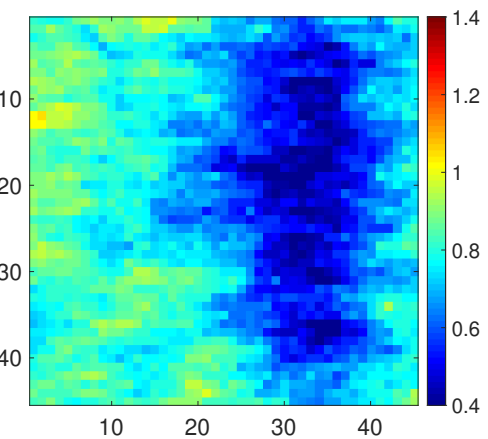

(h) STD - both

Fig. 14: The means and standard deviations of the initial ensemble and the updated ensembles of logpermeability after the assimilation of saturation fronts at day 60 for the three cases. 


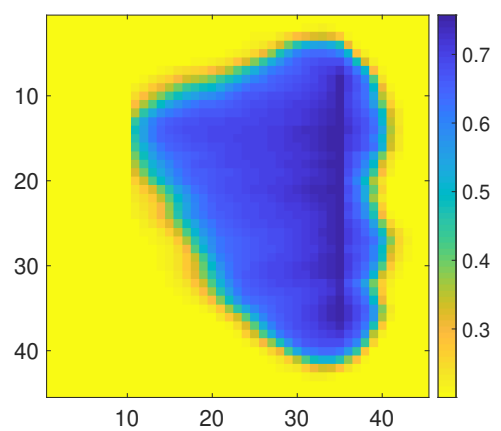

(a) Mean - EM only

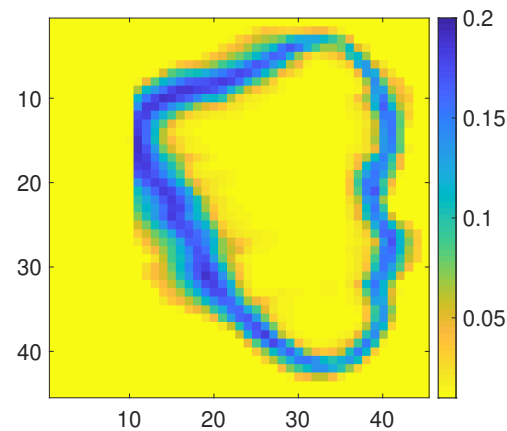

(d) STD - EM only

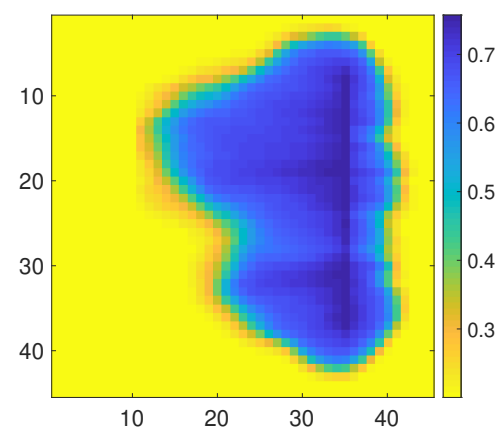

(b) Mean - seismic only

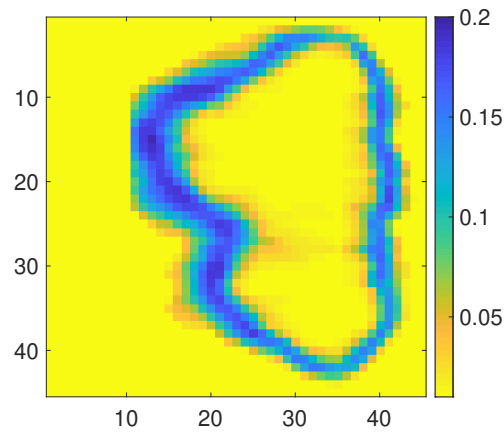

(e) STD - seismic only

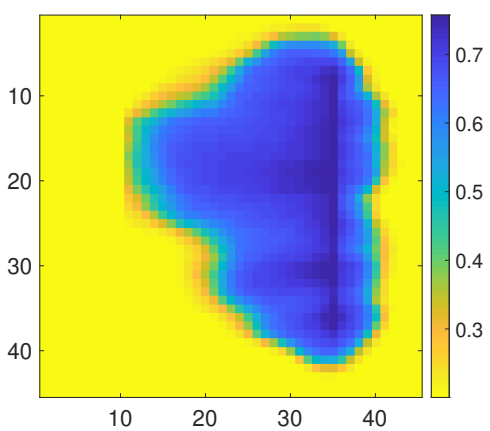

(c) Mean - both

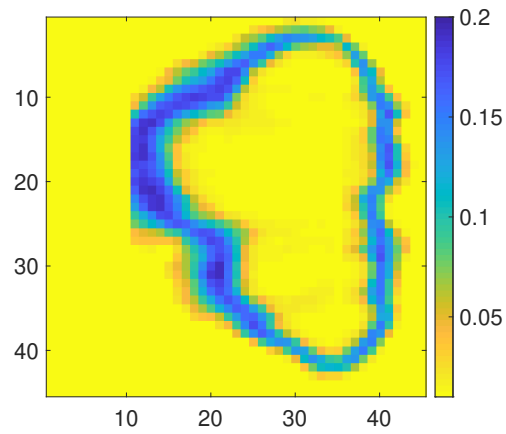

(f) STD - both

Fig. 15: The means and standard deviations of the prior ensembles of water saturation at day 180 for the three cases.

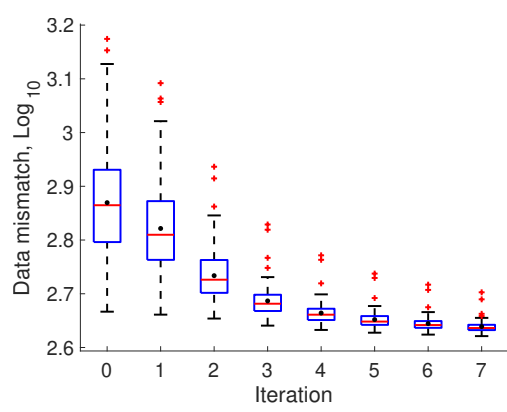

(a) EM only

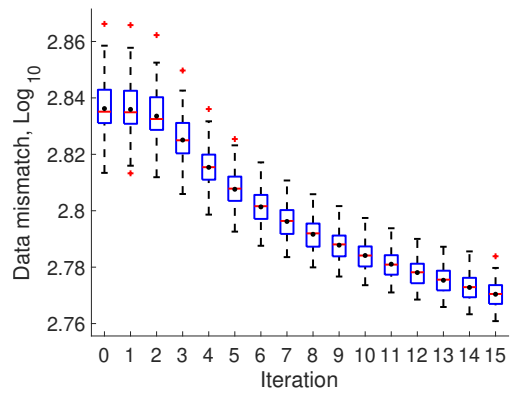

(b) Seismic only

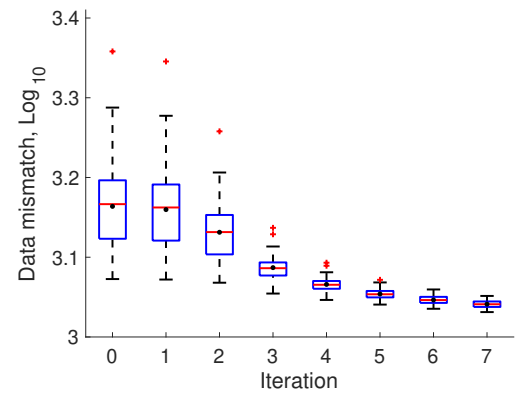

(c) Both

Fig. 16: Boxplots of data mismatch as functions of iteration step for separate and joint assimilation of seismic and EM data at day 180. 
Fig. 17 compares the distributions of the RMSEs of porosity and water saturation along with the iterations for the three cases. In the EM only case, the RMSE of water saturation is reduced consistently but the RMSE of porosity is hardly reduced. In the seismic only case, the RMSE of porosity starts to increase after the $4^{\text {th }}$ iteration while the RMSE of water saturation almost stays at the same level. This indicates that overfitting of the data might occur. Considering the fact that we implement a relatively strong localization in all cases, we look back at the initial data mismatch, which is found to be already rather small, approximately half the number of seismic data. It means that we are actually matching the observation noise. The overfitting issue also reflects the lesser sensitivity of seismic data to saturation changes in contrast to EM data. The joint assimilation of seismic and EM data leads to the best result in which the RMSEs of porosity and water saturation are both consistently reduced, however, at different degrees. The improvement on the estimation of water saturation is dominant at this integration step, and the updated ensemble means of saturation for the three cases are shown in Fig. 18.

Day 180 - step 2. We continue to the second integration step, but only with the case of joint incorporation of seismic and EM data considering its obvious superiority. Fig. 19 illustrates the extraction of saturation front from the updated ensemble mean in the same way as before. The extracted saturation front (in red dots, with a threshold value of 0.3 ) is shown to be in good agreement with the reference one (in greed circles). The standard deviation of one grid cell length is used for the observation errors, which is roughly estimated according to the distribution of the extracted fronts from the updated saturation ensemble as shown in Fig. 19(c).

Fig. 20 shows a consistent reduction of both data mismatch and the RMSE of logpermeability along with the iterations. The mean and standard deviation of the predicted saturation ensemble at day 180 after the second integration step is shown in Fig. 21, indicating a close match to the interpreted front.

Fig. 22 presents the means and standard deviations of the final porosity and permeability ensembles after the whole history matching. The improvement in the permeability 


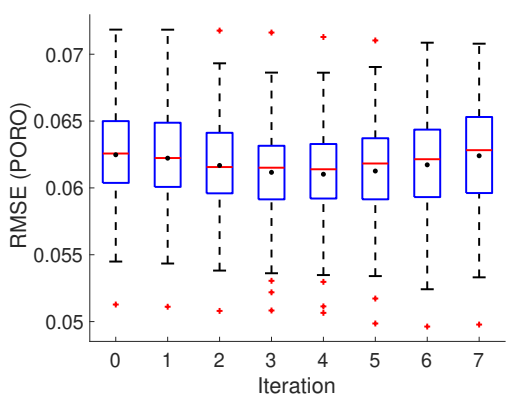

(a) EM only - porosity

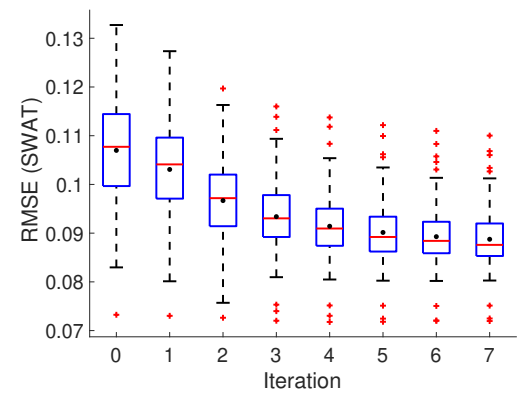

(d) EM only - saturation

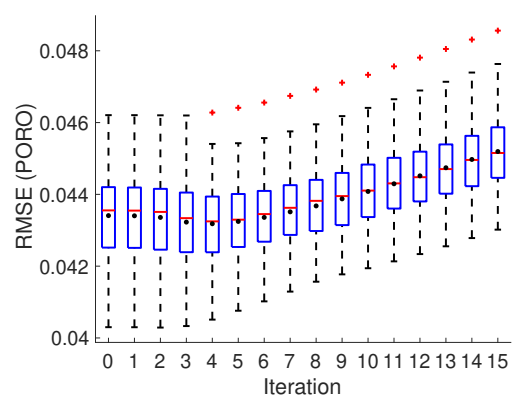

(b) Seismic only - porosity

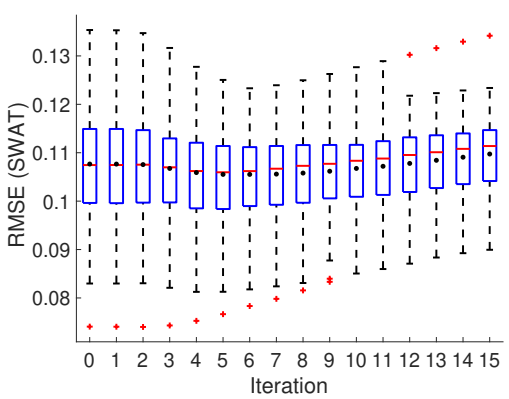

(e) Seismic only - saturation

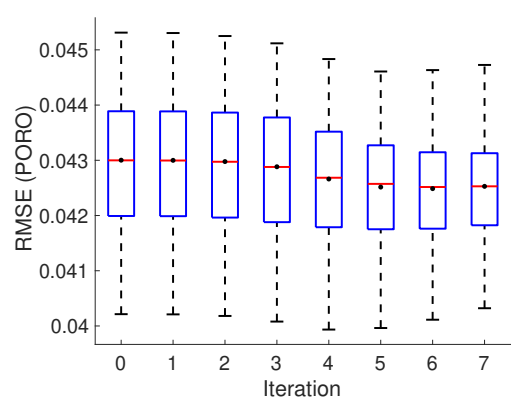

(c) Both - porosity

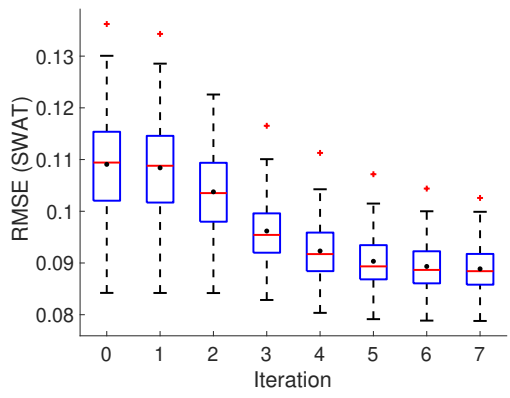

(f) Both - saturation

Fig. 17: Boxplots of RMSEs of porosity and water saturation as functions of iteration step for separate and joint assimilation of seismic and EM data at day 180 .

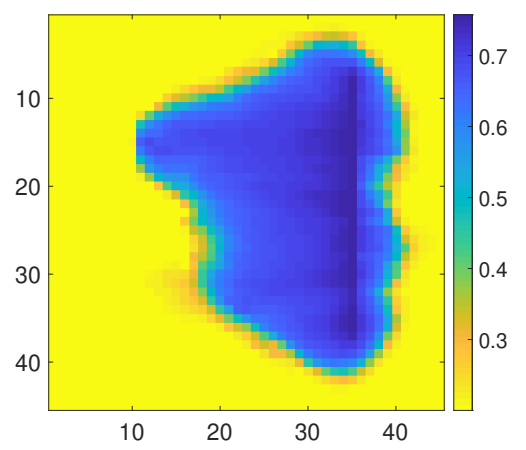

(a) EM only

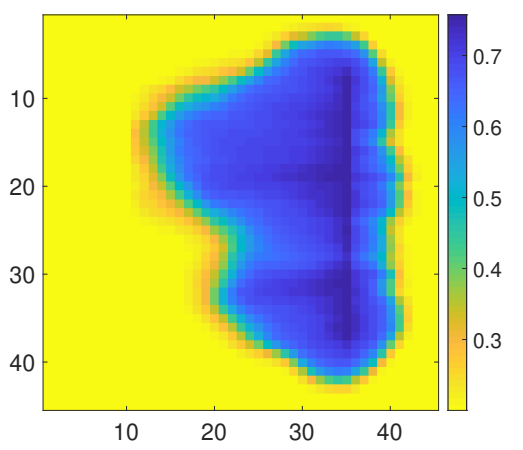

(b) Seis only

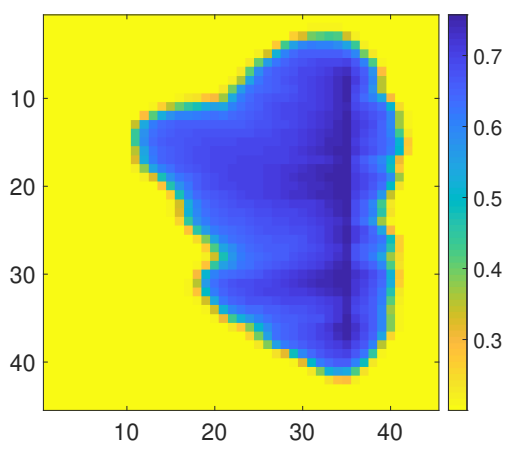

(c) Both

Fig. 18: The means of water saturation for the updated ensembles after separate and joint assimilation of seismic and EM data at day 180. 


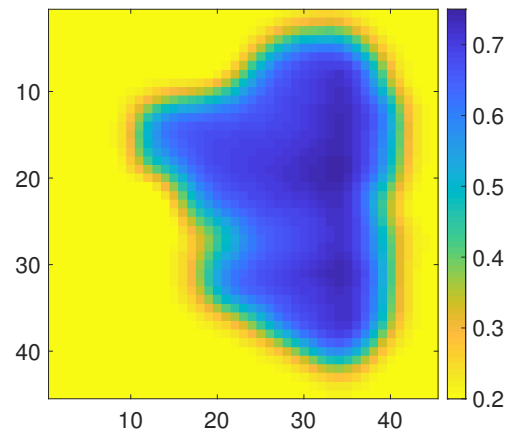

(a) Smoothed mean

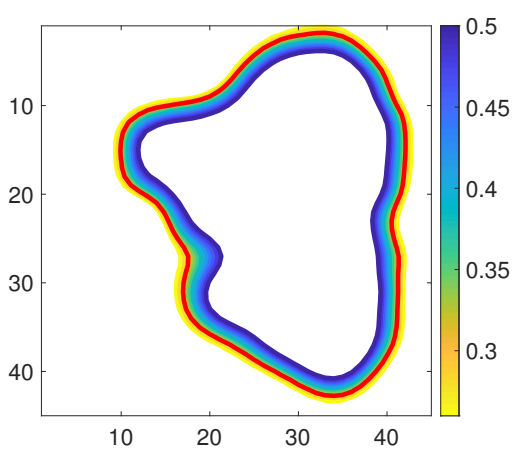

(b) Truncated contours

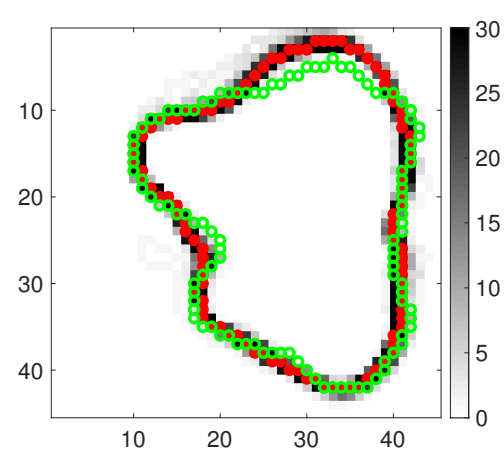

(c) Extracted fronts

Fig. 19: Determination and extraction of saturation fronts from the inverted mean saturation field at day 180. The red isoline in (b) corresponding to 0.3 indicates the interpreted front selected for history matching. Other setting is the same as in Fig. 11.
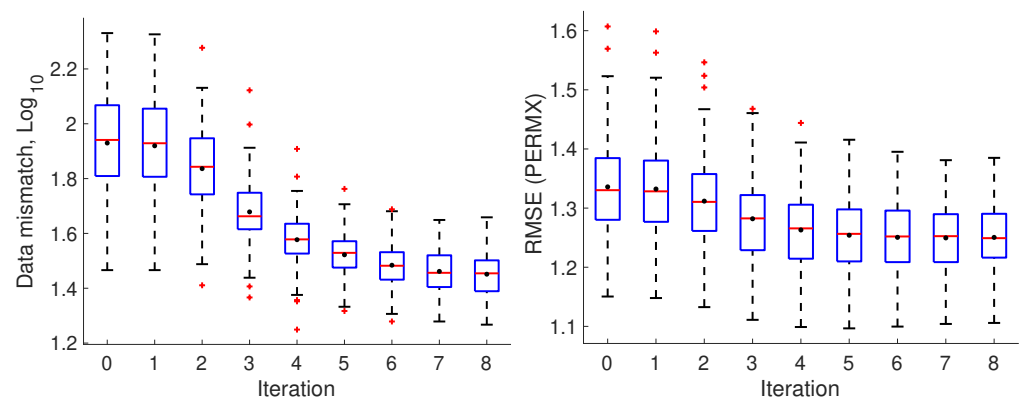

Fig. 20: Boxplots of data mismatch and the RMSE of log-permeability as functions of iteration step after the assimilation of saturation fronts at day 180 .

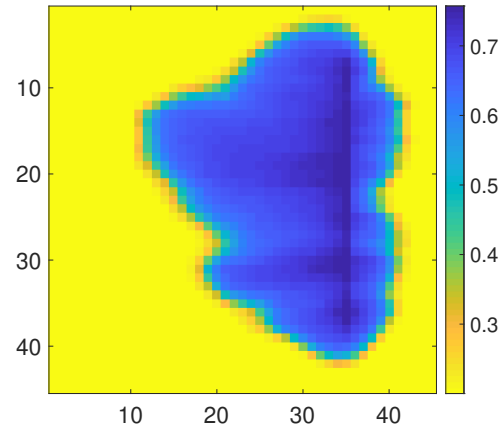

(a) Mean

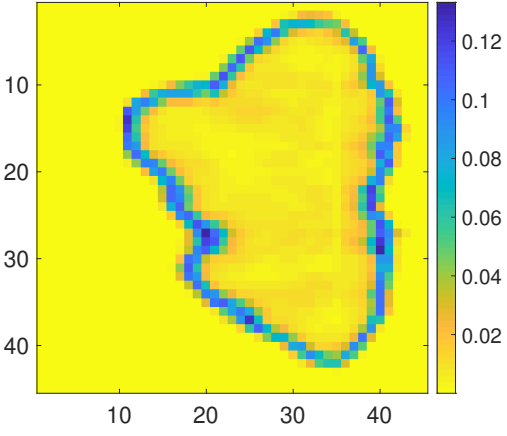

(b) STD

Fig. 21: The mean and standard deviation of the predicted ensemble of water saturation at day 180 after the second assimilation step. 
field is distinct as the top long curvy channel structure is further strengthened and the middle short channel structure is vaguely reproduced. The change in the porosity field is minor due to the strong update at the first assimilation time, from which the major channel features resembling the ones in the reference field are already reproduced. Because we only utilize the front information instead of the whole inverted saturation field for better computational efficiency and stability, we may lose the match to the data at a certain degree. Fig. 23 shows the resulting loss on the data match from this process. The final data mismatch increases only slightly compared with the intermediate result obtained after the first integration step at each assimilation time, which reflects the efficiency of the feature-oriented approach for the joint integration of seismic and EM data.

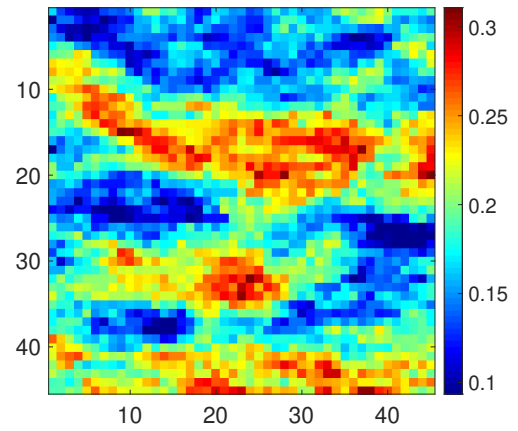

(a) Mean - porosity

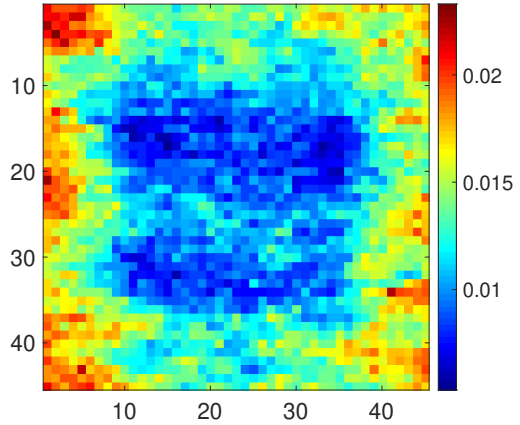

(c) STD - porosity

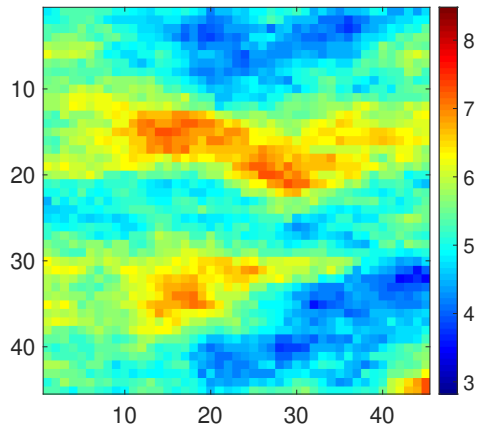

(b) Mean - permeability

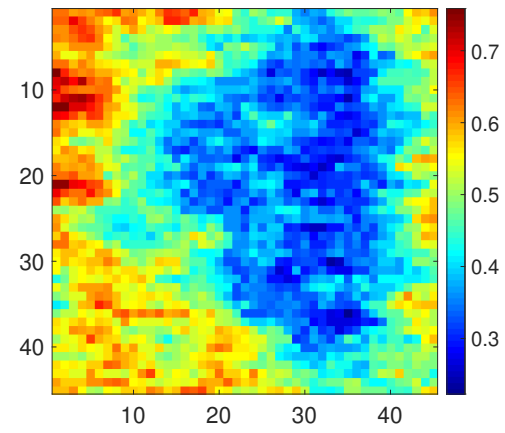

(d) STD - permeability

Fig. 22: The means and standard deviations of the final porosity and log-permeability ensembles after history matching. 


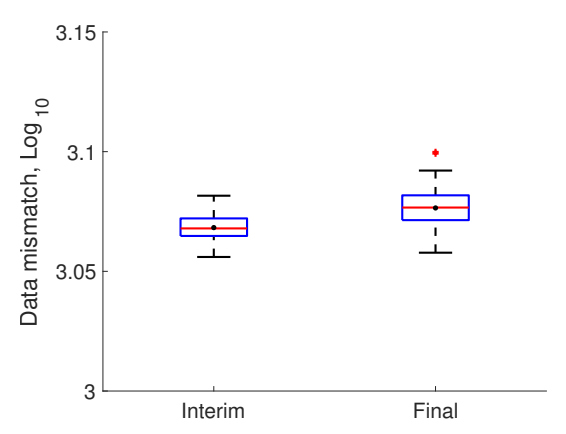

(a) Day 60

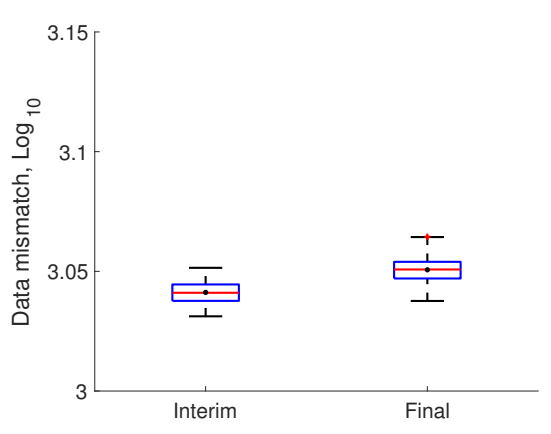

(b) Day 180

Fig. 23: Evaluation of seismic and EM data match loss from the second integration step during history matching. The label interim represents the intermediate data mismatch after the first integration step at each assimilation time corresponding to the data mismatch at the final iteration in Figs. 7(c) and 16(c) . The label final denotes the data mismatch for the final updated ensemble of porosity and permeability.

\subsubsection{Case II: Retain porosity-permeability dependence}

In the above experiments, the omission of the dependence between the porosity and permeability distributions during assimilation leads to a certain loss of the cross-correlations well captured by the initial ensemble. In such situation, one can retain the porositypermeability correlations via Eq. (8) at the second integration step using the ensemble methods without iterations, i.e,

$$
\mathbf{M}_{\kappa}^{a}=\mathbf{M}_{\kappa}^{f}+\Delta \mathbf{M}_{\kappa}^{f} \Delta \mathbf{M}_{\phi}^{f \mathrm{~T}}\left[\Delta \mathbf{M}_{\phi}^{f} \Delta \mathbf{M}_{\phi}^{f \mathrm{~T}}+\Delta \mathbf{M}_{\phi}^{a} \Delta \mathbf{M}_{\phi}^{a \mathrm{~T}}\right]^{-1}\left(\mathbf{M}_{\phi}^{a}-\mathbf{M}_{\phi}^{f}\right)
$$

where $\mathbf{M}_{\kappa}$ and $\mathbf{M}_{\phi}$ are the ensembles of permeability and porosity, respectively, with the superscript $f$ denoting the prior and the superscript $a$ denoting the posterior. Fig. 24 shows the result of conditioning the permeability ensemble on the updated porosity ensemble from the joint assimilation of seismic and EM data at day 60. In principle, localization should be applied to reduce the influence of sampling errors due to the small ensemble size, but for demonstration purpose, no localization is used here, which partly explains the significant reduction of the posterior standard deviation as shown in Fig. 24(b). Obviously, compared with the previous result as shown in Fig. 22, the updated ensemble of permeability gains better characterization (especially the bottom channel structure that is not well reflected by the saturation changes) and shows stronger correlations with the 
updated ensemble of porosity. The result indicates that it is necessary to retain the dependence of associated model variables when the cross-correlations between these variables are strong and properly characterized by the initial sampling.

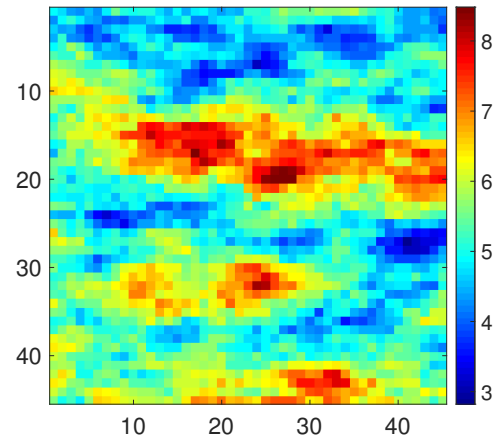

(a) Mean

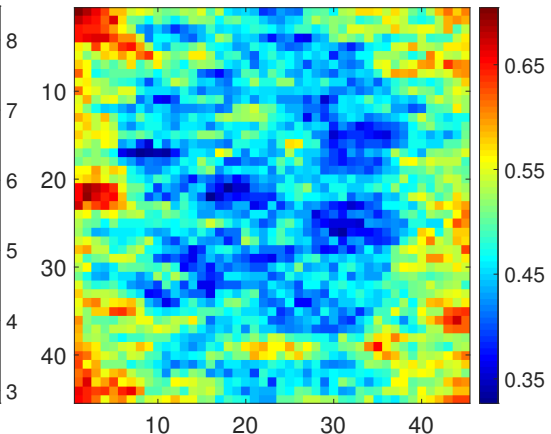

(b) STD

Fig. 24: The mean and STD of the updated ensemble of permeability obtained after conditioning on the updated ensemble of porosity field from the joint assimilation of seismic and EM data at day 60 .

\subsubsection{Case III: Improper initial sampling}

Spurious correlations and biases can propagate or be introduced if the initial ensemble fails to represent the cross-correlations properly. As a poof of concept, we create a new test case by simply replacing the reference porosity field in Fig. 4(a) with the one in Fig. 25(a) (all the other settings remain the same as before). The channelized features are removed from the new reference porosity field. By doing so, we purposely make the original initial ensemble wrongly quantify the true porosity-permeability correlations in the new case. Fig. 25(b) shows the reference saturation field at day 60 under the new setting.

For comparison, we history match seismic and EM data at day 60 using two different approaches. In the first approach, we update the porosity and permeability distributions separately using the proposed two-step integration workflow but ignore the dependence between porosity and permeability (i.e., without implementing Eq. (23) at step 2). In the second approach, we update the porosity and permeability distributions simultaneously with a direct integration of seismic and EM data using the LM-EnRML. This corresponds to the integration at Level 1 in Fig. 1, which is the most straightforward way for joint 


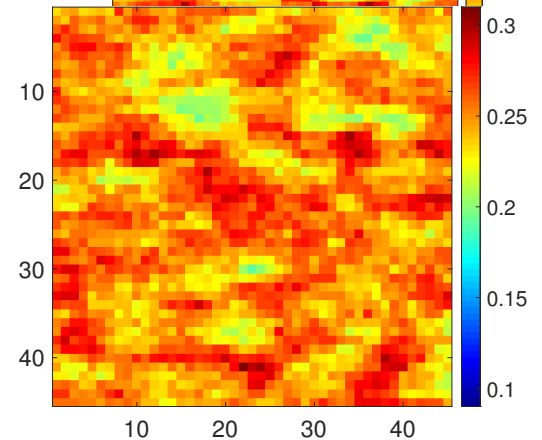

(a) Reference porosity

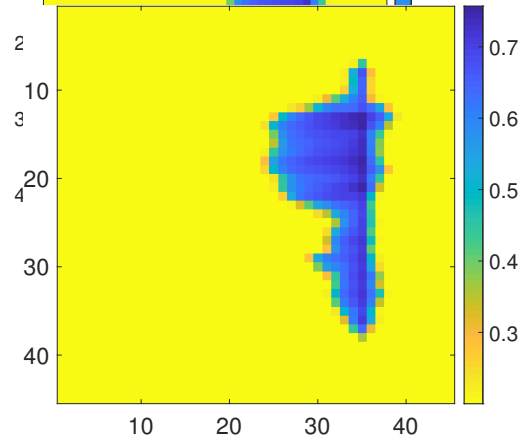

(b) Reference saturation

Fig. 25: Reference porosity and saturation fields at day 60 .

data assimilation without any inversion process involved. Because the cross-correlation between porosity and permeability captured by the initial ensemble is propagated implicitly through the corresponding sample covariances of these variables with the observations (i.e., the term $\Delta \mathbf{M}^{\ell} \Delta \mathbf{D}^{\ell \mathrm{T}}$ in Eq. (14)), the second approach is expected to perform similarly as the first approach but with the conditioning step of Eq. (23). Therefore, by comparing these two approaches, we aim to analyze the impact of the decoupling of associated model variables on the model update in the case of improper initial sampling.

Fig. 26 shows the distributions of data mismatch and the RMSEs of the updated ensembles of model variables along with the iterations for the first (at step 1) and the second approaches. The two approaches give very close performance in terms of the reduction of data mismatch and the RMSE of porosity. The slight increase of the RMSE of porosity at later iterations suggests that either an early termination of the iteration process or a stronger localization on the porosity update should have been adopted. Note that the same localization factor $\gamma=0.3$ was initially applied to all model variables in each approach. However, for both saturation (in approach I) and permeability (in approach II), anticipated negative effect was observed that the corresponding RMSE of the updated ensemble deteriorated at a later stage of the iterations (especially for the updated permeability ensemble whose distribution of RMSEs along with the iterations took on a V-shape). To mitigate this negative effect caused by the improper initial sampling of the porosity distribution, a stronger localization with $\gamma=0.15$ for both saturation and 
permeability is then implemented, which leads to a better result as shown in Fig. 26. Still, there is a little increase in the RMSE of permeability at later iterations due to the strong spurious porosity-permeability correlations introduced by the ill-specified initial ensemble.
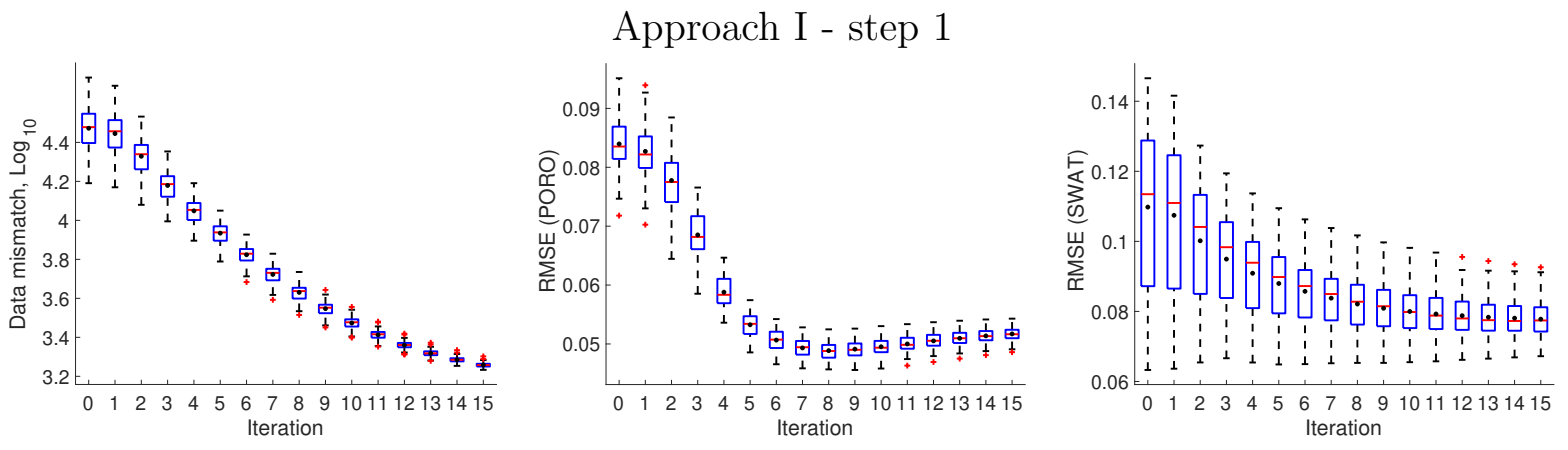

Approach II
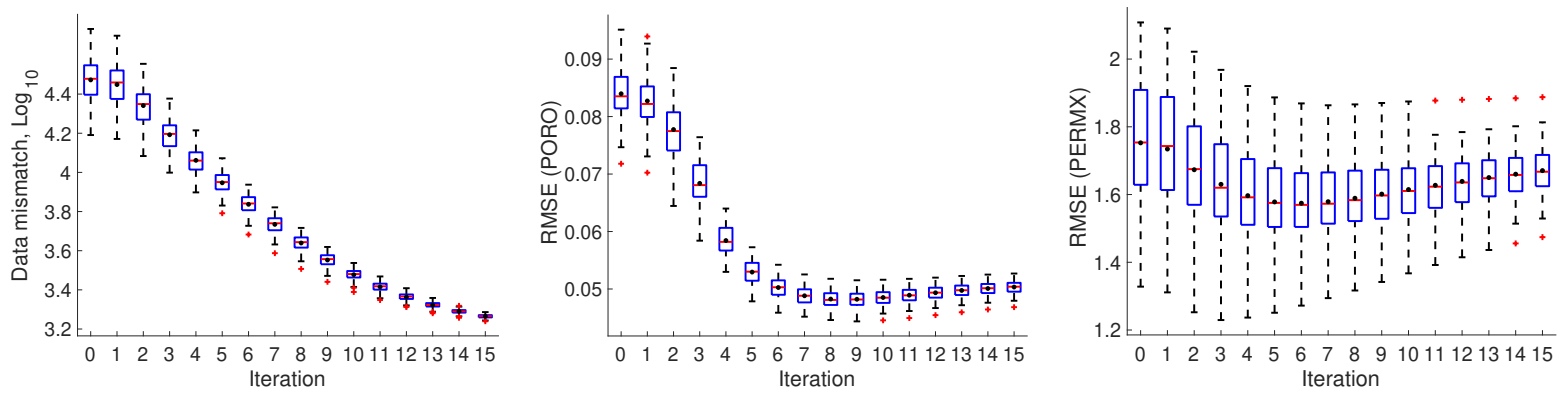

Fig. 26: Boxplots of data mismatch and the RMSEs of the updated ensembles of model variables as functions of iteration step for the first (at step 1) and the second approaches.

Fig. 27 illustrates the determination and extraction of saturation fronts from the inverted mean saturation field at day 60 for the first approach at step 2. As expected, the improper initial sampling limits the performance of the updated ensemble mean (as in Fig. 27(a)) in reproducing important features observed in the reference saturation field. The extracted saturation front (red contour in Fig. 27(b)) to be assimilated at step 2 is determined by a threshold value of 0.48 . This threshold value is selected according to the unstable transition area marked by black rectangle. Fig. 27(c) displays the distribution of extracted fronts from the updated saturation ensemble, which indicates a relatively poor match to the corresponding reference saturation front. The standard deviation of observation error is assumed to be one grid cell length. Fig. 28 shows the distributions 
of the data mismatch and the RMSE of permeability during the assimilation at step 2 . Compared with the final RMSE of permeability from the second approach in Fig. 26, the one from the first approach is further reduced significantly.

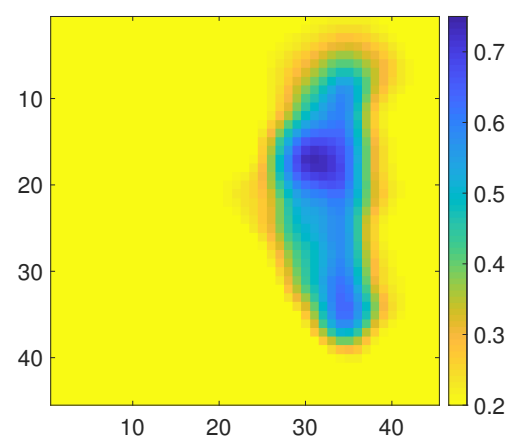

(a) Smoothed mean

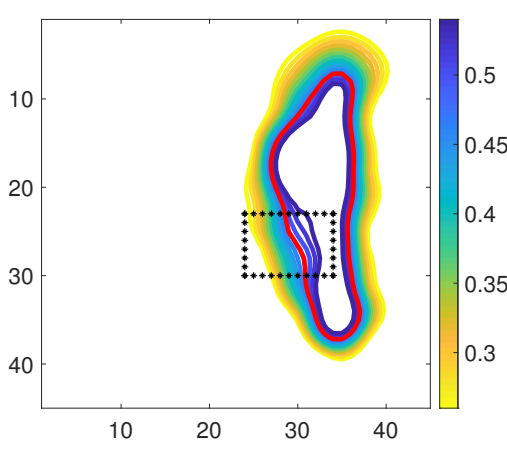

(b) Truncated contours

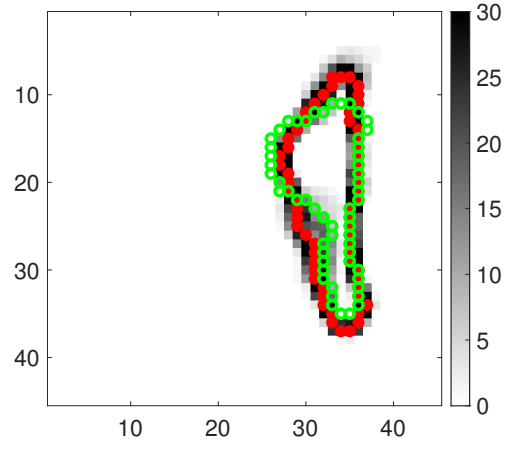

(c) Extracted fronts

Fig. 27: Determination and extraction of saturation fronts from the inverted mean saturation field at day 60. The red isoline in (b) corresponding to 0.48 indicates the interpreted front selected for history matching. Other setting is the same as in Fig. 11.
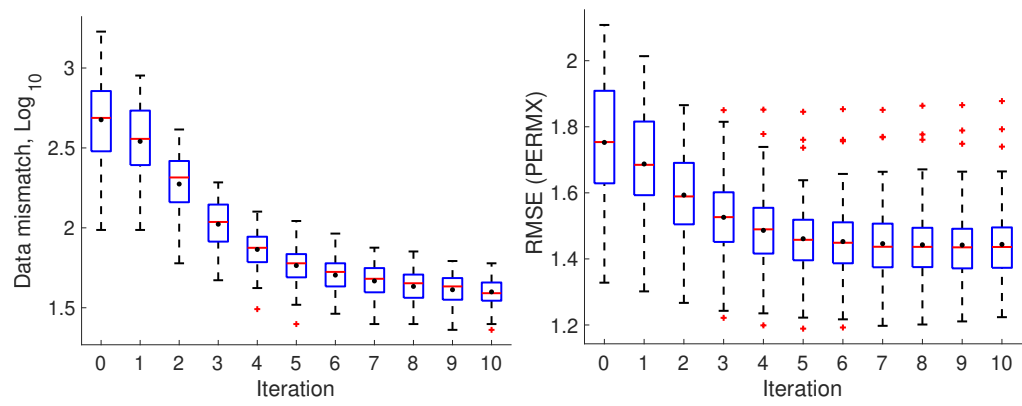

Fig. 28: Boxplots of data mismatch and the RMSE of log-permeability as functions of iteration step after the assimilation of saturation fronts at day 60 .

Fig. 29 summarizes the final updated ensembles of model variables for the two approaches, in which the ones for water saturation are obtained by rerunning the forward flow simulation using the final updated ensembles of porosity and permeability. By comparison, the first approach outperforms the second approach in the characterization of permeability field. It benefits from the separate updating scheme and the omission of the ill-specified cross-correlations between porosity and permeability fields. Even though the second approach imposes a strong localization on the update of permeability, the adverse 
effect caused by spurious correlations remains significant as implied in Fig. 29(h). As to the predicted distribution of water saturation, however, the second approach gives a better match to the reference saturation field. This seems understandable considering the fact that the saturation distribution is updated indirectly in the second approach, thus suffering less from the influence of the improper initial sampling compared with the first approach. In general, the first approach yields more robustness to the initial sampling errors. As already stressed in Section 2.1, the results also verify the fact that sampling the initial ensemble appropriately is fundamental to ensure the performance of ensemble-based data-assimilation methods. More importantly, the comparative analysis of the influence of the decoupling on the model update offers a way for the diagnosis of improper initial sampling.

\subsection{Realistic 3D reservoir model}

To further examine the performance of the proposed workflow in a more realistic setting, we consider an open-source synthetic reservoir model and associated data set published by Jansen et al. [26].

Experimental setting. The model has dimensions of $60 \times 60 \times 7$ with 18553 active grid cells. The grid-cell size is $8 \mathrm{~m}$ in the $x$ - and $y$-direction and $4 \mathrm{~m}$ in the $z$-direction. The fluid system consists of two immiscible phases, oil and water. There are 12 vertical wells completed in all layers, including 8 water injectors and 4 oil producers as shown in Fig. 30. The injectors are controlled by a constant injection rate of $49.5 \mathrm{sm}^{3} / \mathrm{d}$, and the producers are controlled by constant bottomhole pressure of 395 bar. For crosswell seismic and EM configurations, transmitters are placed in the injector I4 located in the middle of the reservoir, and receivers are placed in the remaining 11 wells. The transmitters and receivers are distributed across the interval of the reservoir with a uniform spacing of 4 m. The data are collected with 9 transmitters and $9 \times 11$ receivers. For the EM survey, the source frequencies are 500 and $1000 \mathrm{~Hz}$. For the seismic survey, frequencies 20, 60 and $100 \mathrm{~Hz}$ are selected for the inversion. The seismic and EM surveys are conducted after 12 months (30 days per month) of production. The total number of seismic and EM data amounts to $8910\left(=9 \times 9 \times 11 \times 2 \times 3^{\text {Seis }}+9 \times 9 \times 11 \times 2 \times 2^{E M}\right)$. The synthetic 


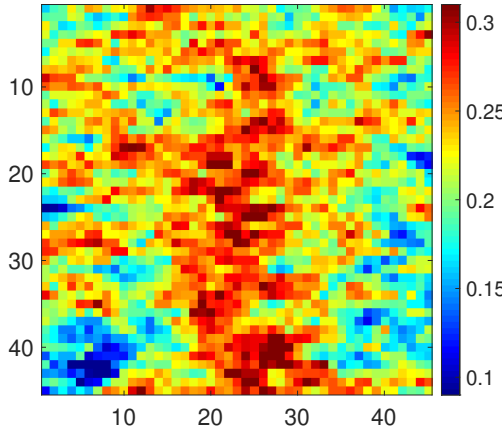

(a) Porosity mean - app. I

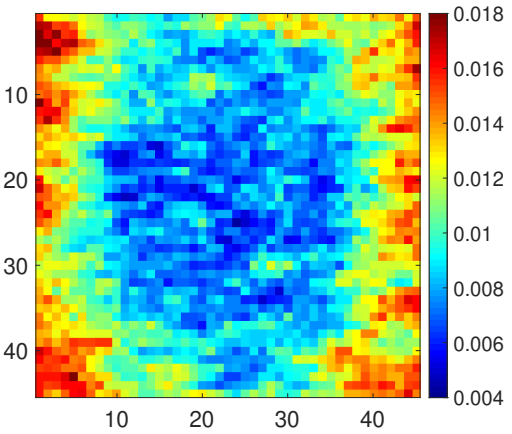

(d) Porosity STD - app. I

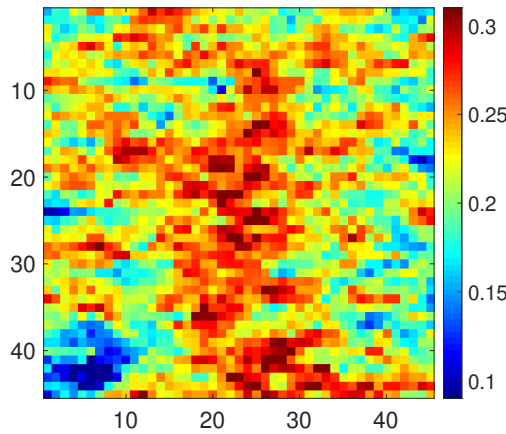

(g) Porosity nean - app. II

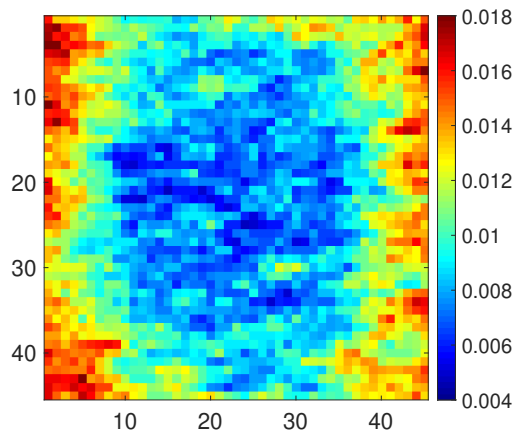

(j) Porosity STD - app. II

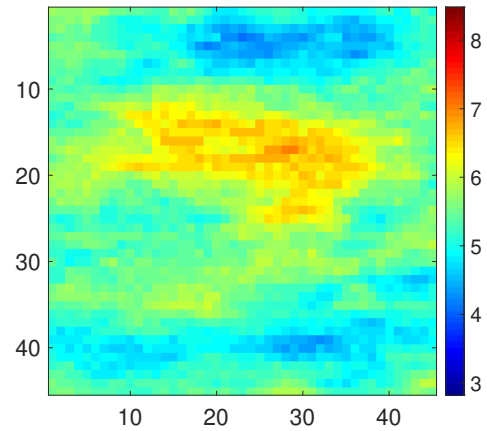

(b) Permeability mean - app. I

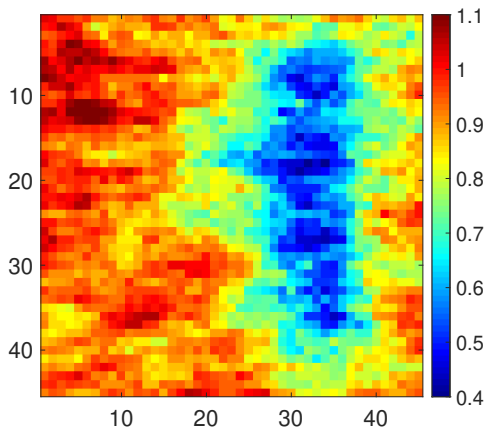

(e) Permeability STD - app. I

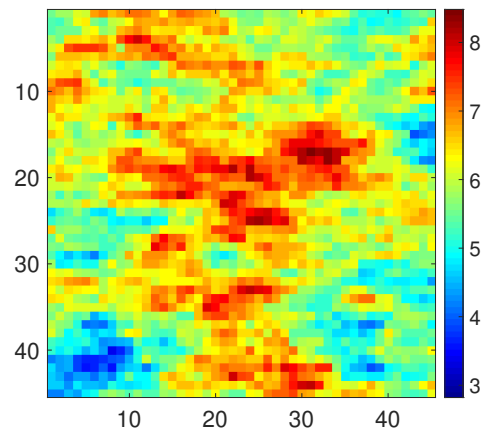

(h) Permeability mean - app. II

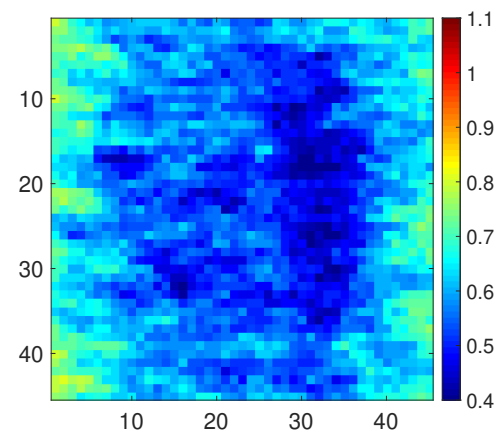

(k) Permeability STD - app. II

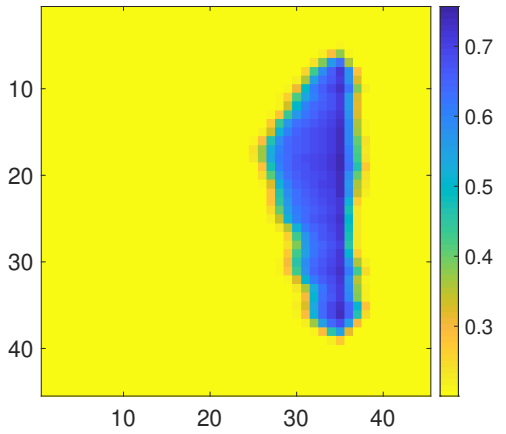

(c) Saturation mean - app. I

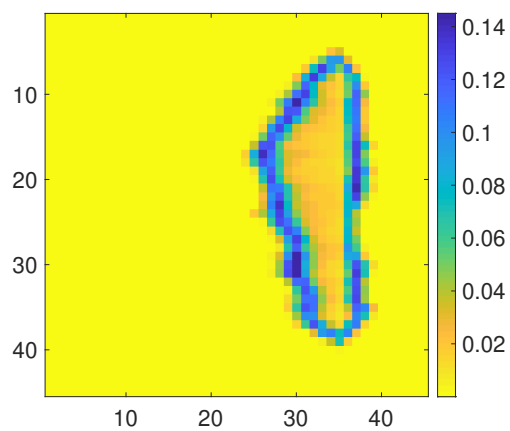

(f) Saturation STD - app. I

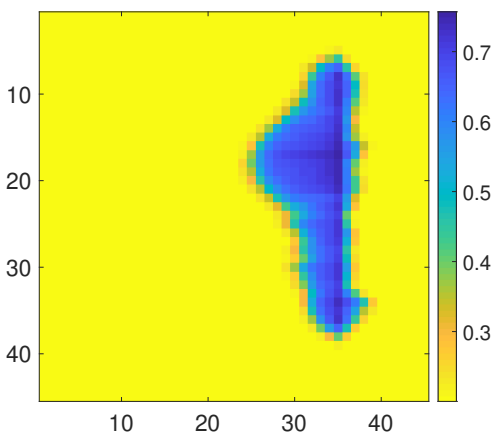

(i) Saturation mean - app. II

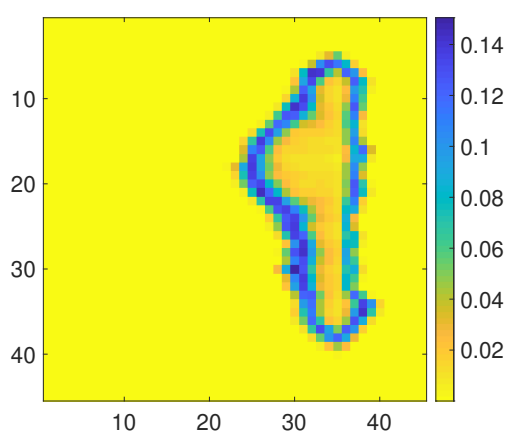

(1) Saturation STD - app. II

Fig. 29: Comparison of final updated ensembles of model variables for the two approaches. 
seismic and EM data are perturbed with 5\% Gaussian random white noise. In addition, well production data are also considered to examine the added value of jointly integrating seismic and EM data on forecasting production. The well production data include waterand oil-production rates of the 4 producers and bottomhole pressures of the 8 injectors, which are collected monthly up to 24 months (resulting in 384 data points totally). The standard deviation of data noise is $5 \mathrm{sm}^{3} / \mathrm{d}$ for the oil-production rate, $8 \mathrm{sm}^{3} / \mathrm{d}$ for the water-production rate, 1 bar for bottomhole pressure. The simulation period consists of 24 months for history matching and 36 months for production forecasting.

The published data set in Jansen et al. [26] contains an ensemble of 101 permeability realizations, based on which we generate the same number of porosity realizations by assuming a stochastic log-linear porosity-permeability relationship. Randomly chosen from these realizations, Fig. 30 shows the reference porosity and log-permeability fields that are used to generate the synthetic data. The remaining 100 realizations of porosity and permeability constitute the initial ensemble. The production data together with seismic-EM data can be history matched either sequentially or simultaneously. In this example, we choose the simultaneous approach given its better performance observed in Zhang et al. [60]. Specifically, production data are assimilated at step 2 of the proposed workflow.

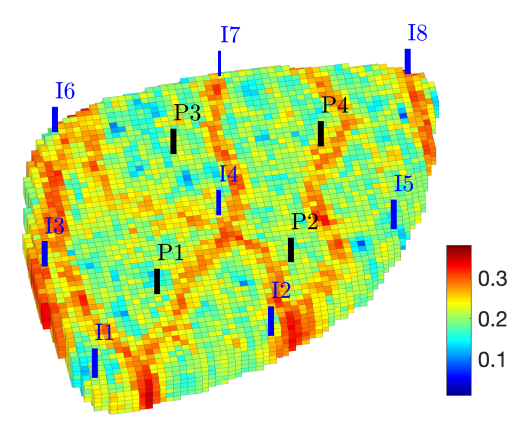

(a) Reference porosity

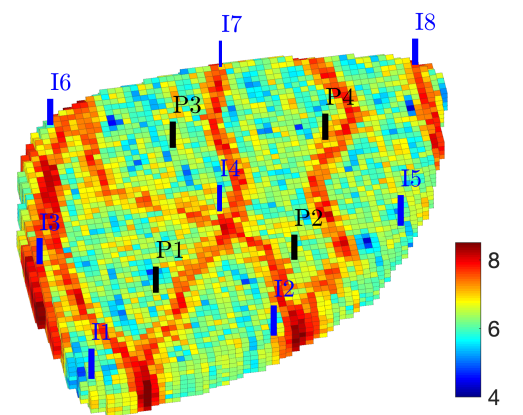

(b) Reference log-permeability

Fig. 30: Reference porosity and log-permeability fields that are used to generate synthetic data. Injecting and producing wells are marked in blue and black colors, respectively.

Results - step 1. Fig. 31 summarizes the result for the joint assimilation of seismic and EM data at step 1. Both data mismatch and the RMSEs of porosity and permeability 
are reduced consistently and significantly with the iterations.
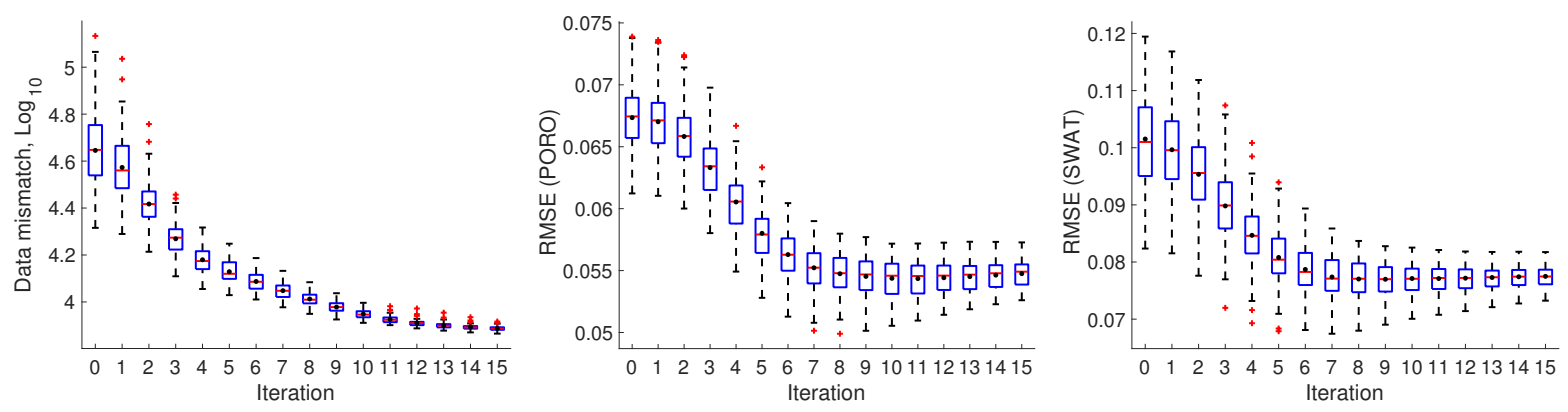

Fig. 31: Boxplots of data mismatch and the RMSEs of the updated ensembles of porosity and saturation as functions of iteration step for joint assimilation of seismic and EM data.

Results - step 2. Even though the inverted saturation fields are 3D, the limited information content of the considered seismic and EM data is not sufficient to give a reliable reproduction of 3D saturation fronts. Therefore, we apply vertical averaging on the inverted saturation fields and extract front positions from the obtained 2D saturation maps. As shown in Fig. 32, the updated mean of saturation maps captures the main features observed in the reference saturation map, and the interpreted fronts distribute closely around the reference front. It is assumed that the interpreted front positions have uncorrelated errors with a standard deviation of one grid cell length.

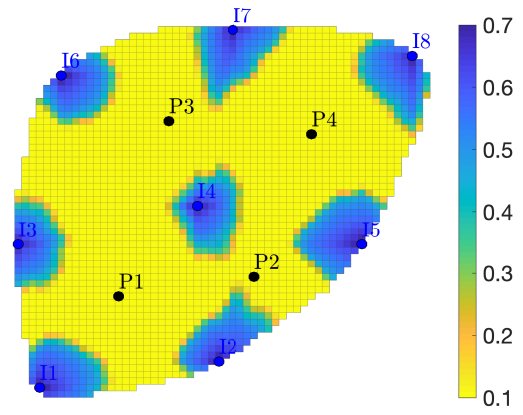

(a) Reference

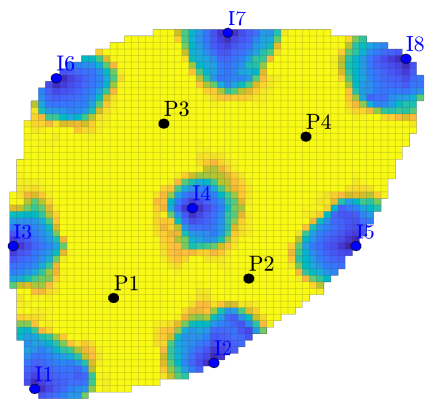

(b) Updated mean

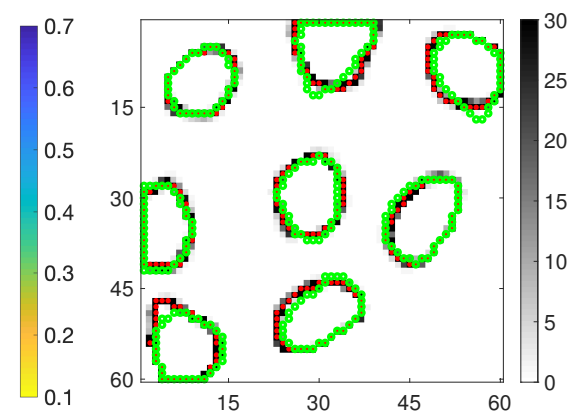

(c) Extracted fronts

Fig. 32: 2D saturation maps from (a) the reference saturation field and (b) the updated mean saturation field obtained by vertical averaging, and (c) the extracted fronts corresponding to a threshold value of 0.2 using the same procedure as in Fig. 11.

Because the strong correlations between porosity and permeability are well captured 
by the initial ensemble, we first condition the permeability ensemble on the updated porosity ensemble using Eq. 23. The Kalman gain localization with $\gamma=1$ is applied to reduce sampling errors. The resulting permeability ensemble is then conditioned on both interpreted saturation fronts and production data, and the updated porosity ensemble from step 1 is conditioned on the production data only. The final updated ensemble means of porosity and permeability after step 2 are shown in Fig. 33 (c) and (d). Compared with the counterparts obtained with production data only in 33 (a) and (b), the integration of seismic and EM data improves the characterization of the considered reservoir properties significantly.

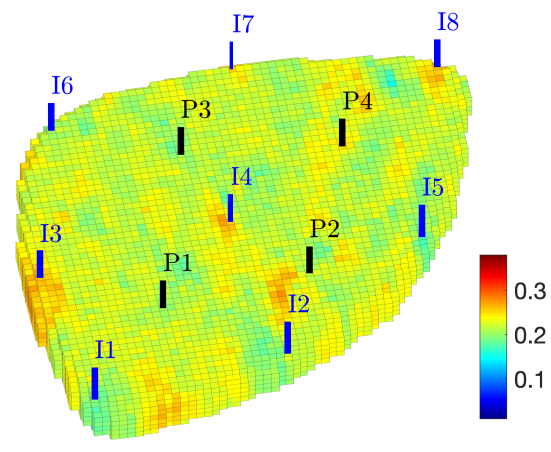

(a) Mean porosity - prodOnly

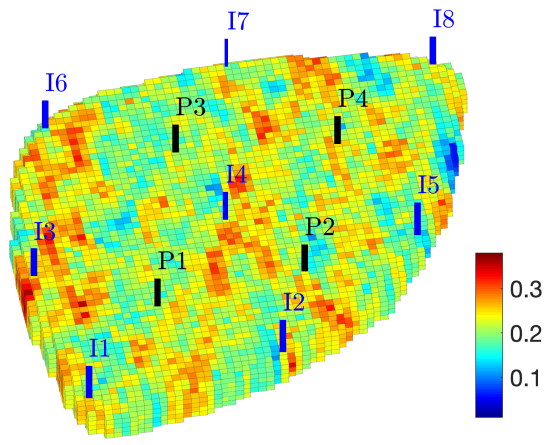

(c) Mean porosity - joint

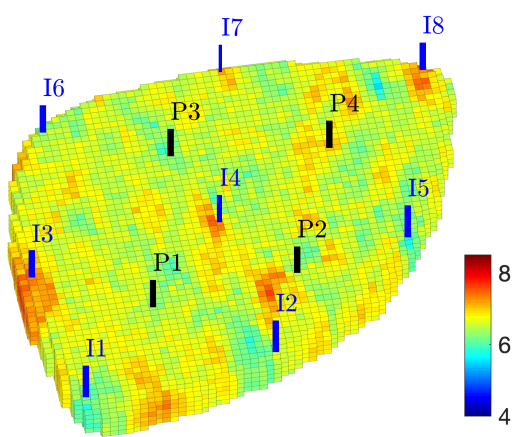

(b) Mean log-permeability - prodOnly

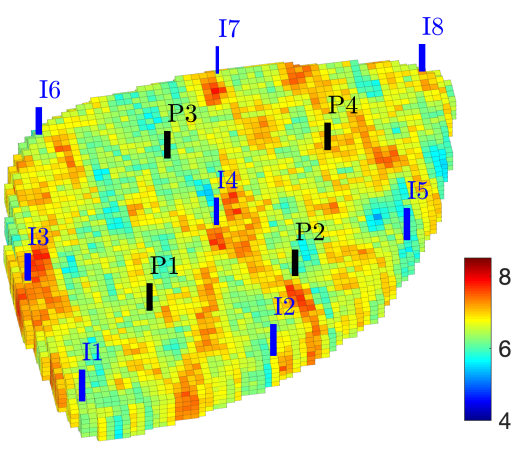

(d) Mean log-permeability - joint

Fig. 33: The means of the final updated porosity and log-permeability ensembles with production data only in (a) and (b), and with joint production-seismic-EM data in (c) and (d).

Fig. 34 shows the match and forecast to production data (in yellow) for the final updated ensemble, which are compared with the ones (in blue) from the updated ensemble conditioned on production data only. The result indicates a positive synergy effect of the 
joint integration on both the match to production data and future forecast.
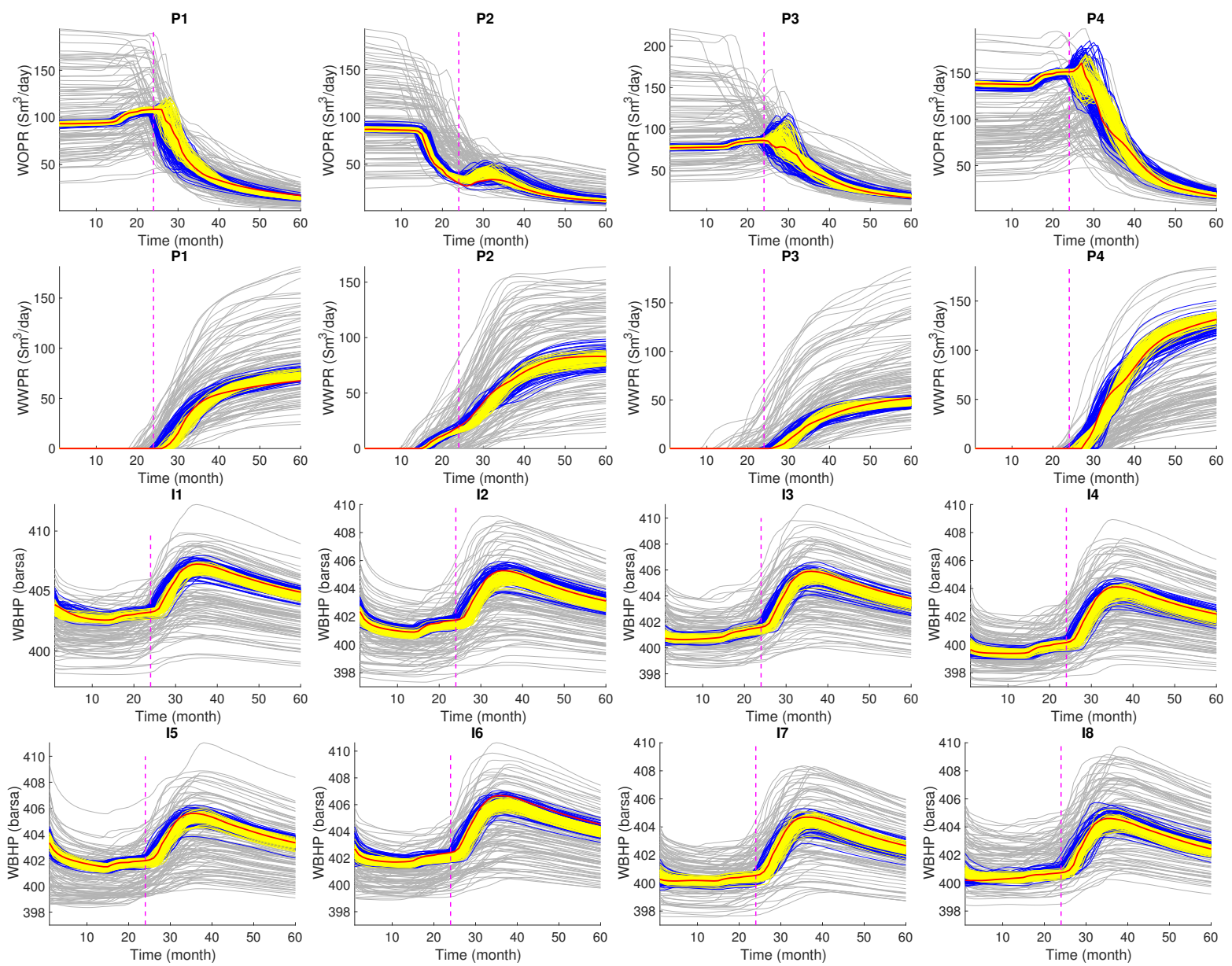

Fig. 34: Production-data match and forecast at all wells for the initial ensemble (gray) and final updated ensembles with production data only (blue), and with joint production-seismic-EM data (yellow). The red line corresponds to the data from the reference model. The history-matching and forecasting periods are separated by the vertical magenta dashed line. WOPR $=$ well oil-production rate; WWPR $=$ well water-production rate; WBHP $=$ well bottomhole pressure.

\section{Discussion and conclusions}

We introduced a feature-oriented ensemble history-matching workflow focusing on the joint assimilation of time-lapse seismic and EM data. The developed workflow divides the history-matching process into two steps. The first step involves a joint inversion of seismic and EM data, from which uncertain reservoir cross-properties such as porosity and 
saturation are updated using ensemble-based assimilation methods. The seismic and EM measurements are typically of high dimensions. This causes difficulties for the ensemblebased methods in which a small ensemble size is usually employed. To deal with that, we adopt an adaptive Kalman gain localization method based on bootstrap sampling. In the second step, the remaining model variables of interest are conditioned on the updated cross-properties. In particular, to assimilate the inverted saturation field efficiently, we take a feature-oriented approach that assimilates the saturation fronts extracted from the inverted saturation field using the ensemble methods with a distance parameterization. Even though the developed workflow focuses only on the assimilation of seismic and EM data, the extension to include other types of measurements is straightforward.

We examined the performance of the developed history-matching workflow using a 2D channelized reservoir model and a more realistic 3D reservoir model with a crosswell configuration for seismic and EM surveys. It was demonstrated that the developed workflow provides a novel and effective way to calibrate reservoir models with multiple sources of geophysical data. The experimental results revealed the complementary nature between seismic and EM data, and showed a positive synergy effect on the characterization of model variables by jointly assimilating both types of data. In the 2D channelized reservoir examples, we showed that the decoupled updating scheme neglecting the dependence of associated model variables was effective in reducing the influence of spurious correlations arising from the improper initial sampling. However, it would suffer from information loss when the correlations between the associated model variables were strong and well represented by the initial ensemble. In particular, the improper initial sampling could cause inconsistent model updates between the cases with and without the decoupling updating scheme. From a practical point of view, this provides a diagnostic tool to assess the validity of the initial sampling.

The promising results under the relatively ideal experimental settings considered in this study still need to be confirmed in real applications. The model error, particularly in the rock physics modeling and observation bias, have been for instance ignored. Besides, simplistic measures (e.g., smoothing and thresholding) have been used for feature 
extraction and quantification of the associated uncertainty. This holds great potential for further improvement by, for instance, exploring more advanced image analysis techniques. More investigation into these aspects is therefore expected to enhance the robustness and efficacy of the developed workflow for history matching.

\section{Acknowledgments}

Support for authors is provided by the research project 'Efficient Integration of Electromagnetic Tomography into Reservoir History Matching' which is funded by Saudi Aramco. The authors also thank Femke C. Vossepoel, Wim Mulder, and Marwan Wirianto for providing the multigrid EM forward solver.

\section{Appendix A Rock physics modeling}

\section{A.1 Petro-elastic model}

The reservoir fluid consists of two phases, oil and water. Gassmann's equations [17] are used to link seismic velocities to reservoir properties, i.e., porosity $\phi$ and saturation. The compressional wave (P-wave) velocity $V_{p}$ in fluid-saturated rocks is expressed as follows:

$$
V_{p}=\sqrt{\frac{K_{s}+\frac{4}{3} \mu_{s}}{\rho_{s}}},
$$

where

$$
\begin{aligned}
& K_{s}=(1-\beta) K_{m}+\beta^{2}\left(\frac{\beta-\phi}{K_{m}}+\frac{\phi}{K_{f}}\right)^{-1}, \\
& K_{f}=\left(\frac{S_{w}}{K_{w}}+\frac{S_{o}}{K_{o}}\right)^{-1}, \\
& \rho_{s}=\phi\left(S_{w} \rho_{w}+S_{o} \rho_{o}\right)+(1-\phi) \rho_{m}, \\
& \beta=\min \left(\frac{\phi}{\phi_{c}}, 1\right) .
\end{aligned}
$$

In the above equations, $K_{s}, \mu_{s}$, and $\rho_{s}$ are saturated-rock bulk modulus, shear modulus, and bulk density, respectively; $K_{m}, K_{o}$, and $K_{w}$ are the bulk moduli of rock matrix, oil, and water, respectively; $\rho_{m}, \rho_{o}$, and $\rho_{w}$ are the densities of rock matrix, oil, and water, 
respectively; $S_{o}$ and $S_{w}$ are oil and water saturations, respectively; $\beta$ is the Biot coefficient which is computed by the critical porosity model of Nur [39] (i.e., Eq. (A.5)), in which $\min (\cdot)$ denotes the minimum operator; and $\phi_{c}$ is the the critical porosity above which solids become suspensions. Here, we use the acoustic approximation so that the shear modulus is neglected.

\section{A.2 Petro-electric model}

Archie's law [5] is used to quantify the relationship of formation conductivity to relevant reservoir properties such as saturation and porosity, which is expressed as follows:

$$
\sigma=a^{-1} \sigma_{w} \phi^{m} S_{w}^{n}
$$

where $\sigma$ and $\sigma_{w}$ are the formation and the brine conductivity, respectively, and $a, m$ and $n$ are Archies parameters whose values normally depend on the rocks cementation, wettability, compaction, pore structure and so on.

Table A.1 summarizes the input parameters of the rock-physics models used in the numerical examples presented in this study.

Table A.1: Input parameters of the rock-physics models.

\begin{tabular}{lllll}
\hline \multicolumn{2}{c}{ Petro-elastic model } & & \multicolumn{2}{c}{ Petro-electric model } \\
\cline { 1 - 2 }$\phi_{c}$ & 0.4 & & $a$ & 1 \\
$\rho_{o}$ & $885 \mathrm{~kg} / \mathrm{m}^{3}$ & & $m$ & 1.5 \\
$\rho_{w}$ & $1050 \mathrm{~kg} / \mathrm{m}^{3}$ & & $n$ & 2 \\
$\rho_{m}$ & $2640 \mathrm{~kg} / \mathrm{m}^{3}$ & & $\sigma_{w}$ & $4.5 \mathrm{~S} / \mathrm{m}$ \\
$K_{m}$ & $35 \mathrm{GPa}$ & & & \\
$K_{w}$ & $2.85 \mathrm{GPa}$ & & & \\
$K_{o}$ & $0.71 \mathrm{GPa}$ & & \\
\hline
\end{tabular}




\section{Appendix B Seismic and EM modeling}

\section{B.1 Seismic forward model}

Full-waveform seismic data are simulated by solving the Helmholtz wave equation in an isotropic and inhomogeneous medium. An acoustic approximation is used as follows:

$$
\nabla \cdot\left[\frac{1}{\rho(\mathbf{r})} \nabla u(\mathbf{r})\right]+\frac{\omega^{2}}{\eta(\mathbf{r})} u(\mathbf{r})=-i \omega q\left(\mathbf{r}_{s}\right),
$$

where $u$ is the pressure field; $\eta$ is the Lamé coefficient related to seismic velocities through the relation $V_{p}=\sqrt{\eta / \rho} ; q$ is the monopole source; $\omega$ is the angular frequency and $i^{2}=$ $-1 ; \mathbf{r}$ denotes spatial positions; $\mathbf{r}_{s}$ denotes source positions; $\nabla$ is the spatial differential operator with respect to $\mathbf{r}$; and · is the vector inner product. The above equation is solved using the frequency-domain finite-difference approach with a perfectly matched layer absorbing boundary condition [45, 49]. Seismic measurements in crosswell setting are defined as a vector of pressure field responses, generated by the sources at positions $\mathbf{r}_{s}$ and observed at receiver locations $\mathbf{r}_{r}$ along boreholes, denoted by

$$
\mathbf{d}_{o b s, \omega_{j}}^{\text {Seis }}=\left[\begin{array}{c}
\boldsymbol{\operatorname { R e }}\left(u_{\mathbf{r}_{s}, \omega_{j}}\left(\mathbf{r}_{r}\right)\right) \\
\boldsymbol{I} \mathbf{\operatorname { m }}\left(u_{\mathbf{r}_{s}, \omega_{j}}\left(\mathbf{r}_{r}\right)\right)
\end{array}\right], \text { for } j=1,2, \ldots, N_{\omega}^{\text {Seis }},
$$

where $\operatorname{Re}(\cdot)$ and $\operatorname{Im}(\cdot)$ denote the operators taking the real and imaginary parts of the argument, respectively. $N_{\omega}^{\text {Seis }}$ is the number of frequencies that are selected for fullwaveform inversion. The total number of seismic measurements at each survey is therefore given by $N_{\mathbf{r}_{s}} \times N_{\mathbf{r}_{r}} \times 2 \times N_{\omega}^{\text {Seis }}$, where $N_{\mathbf{r}_{s}}$ and $N_{\mathbf{r}_{r}}$ are the numbers of the source locations and the receiver locations, respectively. For full-waveform seismic data, a frequencyweighting scheme introduced by Hu et al. [23] is used,

$$
\mathbf{d}_{o b s, \omega_{j}}^{\text {Seis }}=\vartheta_{j} \cdot \mathbf{d}_{o b s, \omega_{j}}^{\text {Seis }}, \text { where } \vartheta_{j}=\frac{\omega_{j}^{-2}}{\sum_{k=1}^{N_{e}^{s e i s}} \omega_{k}^{-2}},
$$

to restrain the high-frequency component from dominating the history-matching process and introducing undesirable artifacts to the estimated reservoir properties. 


\section{B.2 EM forward model}

The EM response is governed by Maxwell's equations. In crosswell setting, an EM survey is normally conducted at only a few low frequencies (more often single frequency), so the problem is usually formulated and solved in the frequency domain. After rearrangement of Maxwell's equations, the second-order electromagnetic equations becomes:

$$
i \omega \mu[\sigma(\mathbf{r})-i \omega \varepsilon] \mathbf{E}(\mathbf{r})-\nabla \times \nabla \times \mathbf{E}(\mathbf{r})=-i \omega \mu \mathbf{J}\left(\mathbf{r}_{s}\right)-\mathbf{M}\left(\mathbf{r}_{s}\right),
$$

where $\mu$ is the magnetic permeability; $\varepsilon$ is the dielectric permitivity; $\nabla \times(\cdot)$ represents the curl operator; $\mathbf{E}$ is the electric field; and $\mathbf{J}$ and $\mathbf{M}$ denote the electric and magnetic current sources, respectively. Perfectly electrically conducting boundary conditions (i.e., $\mathbf{n} \times \mathbf{E}=$ 0 ) are imposed, where $\mathbf{n}$ is the outward normal on the boundary of the domain. With the input of $\sigma(\mathbf{r})$ provided by the rock physics model in Eq. (A.6), the resulting system of equations above is solved with a 3D multigrid solver described in $[38,47]$. Once the electric field $\mathbf{E}$ is known, the magnetic field can be obtained from $\mathbf{H}(\mathbf{r})=(i \omega \mu)^{-1} \nabla \times \mathbf{E}(\mathbf{r})$. The crosswell EM measurements are defined as a vector of magnetic field responses, generated by magnetic current source at positions $\mathbf{r}_{s}$ and observed at receiver locations $\mathbf{r}_{r}$ along boreholes, denoted by

$$
\mathbf{d}_{o b s, \omega_{j}}^{E M}=\left[\begin{array}{c}
\boldsymbol{R e}\left(\mathbf{H}_{\mathbf{r}_{s}, \omega_{j}}\left(\mathbf{r}_{r}\right)\right) \\
\mathbf{I m}\left(\mathbf{H}_{\mathbf{r}_{s}, \omega_{j}}\left(\mathbf{r}_{r}\right)\right)
\end{array}\right], \text { for } j=1,2, \ldots, N_{\omega}^{E M},
$$

where the subscript $\omega_{j}$ indicates that the EM measurements are frequency-dependent and $N_{\omega}^{E M}$ is the number of frequencies at which a crosswell EM survey is conducted. The total number of EM measurements at each survey is $N_{\mathbf{r}_{s}} \times N_{\mathbf{r}_{r}} \times 2 \times N_{\omega}^{E M}$.

\section{References}

[1] Aanonsen, S.I., Nævdal, G., Oliver, D.S., Reynolds, A.C., Vallès, B., 2009. Ensemble Kalman filter in reservoir engineering - a review. SPE Journal 14, 393-412. doi:10. 2118/117274-PA. 
[2] Abubakar, A., Gao, G., Habashy, T.M., Liu, J., 2012. Joint inversion approaches for geophysical electromagnetic and elastic full-waveform data. Inverse Problems 28, 055016. doi:10.1088/0266-5611/28/5/055016.

[3] Ait-El-Fquih, B., El Gharamti, M., Hoteit, I., 2016. A bayesian consistent dual ensemble Kalman filter for state-parameter estimation in subsurface hydrology. Hydrology and Earth System Sciences 20, 3289-3307. doi:10.5194/hess-20-3289-2016.

[4] Alfonzo, M., Oliver, D.S., 2019. Seismic data assimilation with an imperfect model. Computational Geosciences , 1-17doi:10.1007/s10596-019-09849-0.

[5] Archie, G.E., 1942. The electrical resistivity log as an aid in determining some reservoir characteristics. Transactions of the AIME 146, 54-62. doi:10.2118/942054-G.

[6] Carlin, B.P., Louis, T.A., 2008. Bayesian methods for data analysis. Chapman and Hall/CRC.

[7] Chen, Y., Oliver, D.S., 2010. Cross-covariances and localization for EnKF in multiphase flow data assimilation. Comput. Geosci. 14, 579-601. doi:10.1007/ s10596-009-9174-6.

[8] Chen, Y., Oliver, D.S., 2013. Levenberg-Marquardt forms of the iterative ensemble smoother for efficient history matching and uncertainty quantification. Computational Geosciences 17, 689-703. doi:10.1007/s10596-013-9351-5.

[9] Chen, Y., Oliver, D.S., 2017. Localization and regularization for iterative ensemble smoothers. Computational Geosciences 21, 13-30. doi:10.1007/ s10596-016-9599-7.

[10] Colombo, D., Rovetta, D., 2018. Coupling strategies in multiparameter geophysical joint inversion. Geophysical Journal International 215, 1171-1184. doi:10.1093/gji/ ggy341. 
[11] Dell'Aversana, P., Bernasconi, G., Miotti, F., Rovetta, D., 2011. Joint inversion of rock properties from sonic, resistivity and density well-log measurements. Geophysical Prospecting 59, 1144-1154. doi:10.1111/j.1365-2478.2011.00996.x.

[12] Deutsch, C.V., 2002. Geostatistical reservoir modeling. Oxford university press.

[13] Emerick, A.A., Reynolds, A.C., 2013. Ensemble smoother with multiple data assimilation. Computers \& Geosciences 55, 3 - 15. doi:10.1016/j.cageo.2012.03.011.

[14] Evensen, G., 2009. Data Assimilation: The Ensemble Kalman Filter. Second ed., Springer Verlag.

[15] Feng, T., Mannseth, T., 2010. Impact of time-lapse seismic data for permeability estimation. Comput. Geosci. 14, 705-719. doi:10.1007/s10596-010-9182-6.

[16] Gao, G., Abubakar, A., Habashy, T.M., 2012. Joint petrophysical inversion of electromagnetic and full-waveform seismic data. Geophysics 77, WA3-WA18. doi:10.1190/geo2011-0157.1.

[17] Gassmann, F., 1951. Elastic waves through a packing of spheres. Geophysics 16, 673-685. doi:10.1190/1.1437718.

[18] Ginting, V., Pereira, F., Rahunanthan, A., 2014. Rapid quantification of uncertainty in permeability and porosity of oil reservoirs for enabling predictive simulation. Mathematics and Computers in Simulation 99, 139-152. doi:10.1016/j . matcom. 2013.04 . 015.

[19] Harris, P., MacGregor, L., 2006. Determination of reservoir properties from the integration of CSEM and seismic data. First Break 24, 53-59.

[20] Hoteit, I., Luo, X., Pham, D.T., 2012. Particle Kalman filtering: A nonlinear bayesian framework for ensemble Kalman filters. Monthly Weather Review 140, 528-542. doi:10.1175/2011MWR3640.1. 
[21] Hoteit, I., Pham, D.T., Gharamti, M., Luo, X., 2015. Mitigating observation perturbation sampling errors in the stochastic EnKF. Monthly Weather Review 143, 2918-2936. doi:10.1175/MWR-D-14-00088.1.

[22] Hoversten, G.M., Cassassuce, F., Gasperikova, E., Newman, G.A., Chen, J., Rubin, Y., Hou, Z., Vasco, D., 2006. Direct reservoir parameter estimation using joint inversion of marine seismic AVA and CSEM data. GEOPHYSICS 71, C1-C13. doi:10.1190/1.2194510.

[23] Hu, W., Abubakar, A., Habashy, T.M., 2009. Simultaneous multifrequency inversion of full-waveform seismic data. Geophysics 74, R1-R14. doi:10.1190/1.3073002.

[24] Iglesias, M.A., 2015. Iterative regularization for ensemble data assimilation in reservoir models. Computational Geosciences 19, 177-212. doi:10.1007/ s10596-014-9456-5.

[25] Jafarpour, B., McLaughlin, D.B., 2009. Reservoir characterization with the discrete Cosine transform. SPE Journal 14, 182-201. doi:10.2118/106453-PA.

[26] Jansen, J.D., Fonseca, R.M., Kahrobaei, S., Siraj, M., Van Essen, G., Van den Hof, P., 2014. The egg model-a geological ensemble for reservoir simulation. Geoscience Data Journal 1, 192-195.

[27] Katterbauer, K., Hoteit, I., Sun, S., 2016. Synergizing crosswell seismic and electromagnetic techniques for enhancing reservoir characterization. SPE Journal 21, 909-927. doi:10.2118/174559-PA.

[28] van Leeuwen, P.J., Evensen, G., 1996. Data assimilation and inverse methods in terms of a probabilistic formulation. Monthly Weather Review 124, 2898-2913. doi:10. 1175/1520-0493(1996)124<2898:DAAIMI>2 . 0 . C0;2.

[29] Leeuwenburgh, O., Arts, R., 2014. Distance parameterization for efficient seismic history matching with the ensemble Kalman filter. Computational Geosciences 18, 535-548. doi:10.1007/s10596-014-9434-y. 
[30] Leeuwenburgh, O., Brouwer, J., Trani, M., 2011. Ensemble-based conditioning of reservoir models to seismic data. Computational Geosciences 15, 359-378. doi:10. $1007 / s 10596-010-9209-z$.

[31] Liang, L., Abubakar, A., Habashy, T.M., 2016. Reservoir property mapping and monitoring from joint inversion of time-lapse seismic, electromagnetic, and production data. Geophysics 81, ID73-ID84. doi:10.1190/geo2015-0620.1.

[32] Lorentzen, R.J., Flornes, K.M., Nævdal, G., 2012. History matching channelized reservoirs using the ensemble Kalman filter. SPE Journal 17, 137-151. doi:10.2118/ 143188-PA.

[33] Luo, X., Bhakta, T., Jakobsen, M., Nævdal, G., 2016. An ensemble 4D-seismic history-matching framework with sparse representation based on wavelet multiresolution analysis. SPE Journal doi:10.2118/180025-PA.

[34] Luo, X., Stordal, A.S., Lorentzen, R.J., Nævdal, G., 2015. Iterative ensemble smoother as an approximate solution to a regularized minimum-average-cost problem: Theory and applications. SPE Journal 20, 962-982. doi:10.2118/176023-PA.

[35] Mavko, G., Mukerji, T., Dvorkin, J., 2009. The rock physics handbook: Tools for seismic analysis of porous media. Cambridge university press.

[36] Moorkamp, M., 2017. Integrating electromagnetic data with other geophysical observations for enhanced imaging of the earth: a tutorial and review. Surveys in Geophysics 38, 935-962. doi:10.1007/s10712-017-9413-7.

[37] Moorkamp, M., Heincke, B., Jegen, M., Roberts, A.W., Hobbs, R.W., 2011. A framework for 3-D joint inversion of MT, gravity and seismic refraction data. Geophysical Journal International 184, 477-493. doi:10.1111/j.1365-246X.2010.04856.x.

[38] Mulder, W., 2006. A multigrid solver for 3D electromagnetic diffusion. Geophysical Prospecting 54, 633-649. doi:10.1111/j.1365-2478.2006.00558.x. 
[39] Nur, A., Mavko, G., Dvorkin, J., Galmudi, D., 1998. Critical porosity: A key to relating physical properties to porosity in rocks. The Leading Edge 17, 3, 357-362.

[40] Obidegwu, D., Chassagne, R., MacBeth, C., 2015. Seismic assisted history matching using binary image matching, in: EUROPEC 2015, Society of Petroleum Engineers.

[41] Oliver, D.S., Alfonzo, M., 2018. Calibration of imperfect models to biased observations. Computational Geosciences 22, 145-161. doi:10.1007/s10596-017-9678-4.

[42] Oliver, D.S., Chen, Y., 2009. Improved initial sampling for the ensemble Kalman filter. Comput. Geosci. 13, 13-27. doi:10.1007/s10596-008-9101-2.

[43] Oliver, D.S., Chen, Y., 2011. Recent progress on reservoir history matching: a review. Comput. Geosci. 15, 185-221. doi:10.1007/s10596-010-9194-2.

[44] Oliver, D.S., Chen, Y., 2018. Data assimilation in truncated plurigaussian models: impact of the truncation map. Mathematical Geosciences 50, 867-893. doi:10.1007/ s11004-018-9753-y.

[45] Operto, S., Virieux, J., Amestoy, P., L'Excellent, J.Y., Giraud, L., Ali, H.B.H., 2007. 3D finite-difference frequency-domain modeling of visco-acoustic wave propagation using a massively parallel direct solver: A feasibility study. Geophysics 72, SM195SM211. doi:10.1190/1.2759835.

[46] Phale, H.A., Oliver, D.S., 2011. Data assimilation using the constrained ensemble Kalman filter. SPE Journal 16, 331-342. doi:10.2118/125101-PA.

[47] Plessix, R.É., Mulder, W.A., 2008. Resistivity imaging with controlled-source electromagnetic data: depth and data weighting. Inverse Problems 24, 034012. doi:10.1088/0266-5611/24/3/034012.

[48] Rovetta, D., Colombo, D., Turkoglu, E., 2017. Petrophysical joint inversion of multi-geophysical attributes and measurements for reservoir characterization, in: SPE Middle East Oil \& Gas Show and Conference, Society of Petroleum Engineers. doi:10.2118/184030-MS. 
[49] Silva, C.D., Herrmann, F., 2019. A unified 2D/3D large-scale software environment for nonlinear inverse problems. ACM Transactions on Mathematical Software (TOMS) 45, 7. doi:10.1145/3291042.

[50] Skjervheim, J.-A., 2007. Continuous updating of a coupled reservoir-seismic model using an ensemble kalman filter technique. The University of Bergen.

[51] Skjervheim, J.A., Evensen, G., Aanonsen, S.I., Ruud, B.O., Johansen, T.A., 2007. Incorporating 4D seismic data in reservoir simulation models using ensemble Kalman filter. SPE Journal 12, 282-292. doi:10.2118/95789-PA.

[52] Strebelle, S., 2002. Conditional simulation of complex geological structures using multiple-point statistics. Math. Geol. 34, 1-21. doi:10.1023/A:1014009426274.

[53] Sun, J., Li, Y., 2016. Joint inversion of multiple geophysical data using guided fuzzy c-means clustering. Geophysics 81, ID37-ID57. doi:10.1190/geo2015-0457.1.

[54] Trani, M., Arts, R., Leeuwenburgh, O., Brouwer, J., 2011. Estimation of changes in saturation and pressure from 4D seismic AVO and time-shift analysis. GEOPHYSICS 76, C1-C17. URL: http://dx.doi.org/10.1190/1.3549756, doi:10. $1190 / 1.3549756$.

[55] Zaki A Al-Ali, Muhammad H Al-Buali, Saleh AlRuwaili, Shouxiang Mark Ma, Alberto F Marsala, David Alumbaugh, Luis DePavia, Cyrille Levesque, Ajay Nalonnil, and Ping Zhang. Looking deep into the reservoir. Oilfield Review, 21(2):38-47, 2009.

[56] Zhang, Y., Leeuwenburgh, O., 2016. Ensemble-based seismic history matching with distance parameterization for complex grids, in: ECMOR XIV-15th European Conference on the Mathematics of Oil Recovery. doi:10.3997/2214-4609.201601815.

[57] Zhang, Y., Leeuwenburgh, O., 2017. Image-oriented distance parameterization for ensemble-based seismic history matching. Computational Geosciences 21, 713-731. doi:10 .1007/s10596-017-9652-1. 
[58] Zhang, Y., Oliver, D.S., 2010. Improving the ensemble estimate of the Kalman gain by bootstrap sampling. Math. Geosci. 42, 327-345. doi:10.1007/s11004-010-9267-8.

[59] Zhang, Y., Oliver, D.S., Chen, Y., Skaug, H.J., 2014. Data assimilation by use of the iterative ensemble smoother for 2D facies models. SPE Journal 20, 169-185. doi:10.2118/170248-PA.

[60] Zhang, Y., Vossepoel, F.C., Hoteit, I., 2020. Efficient Assimilation of Crosswell Electromagnetic Data Using an Ensemble-Based History-Matching Framework. SPE Journal 25, 119-138. doi:10.2118/193808-PA. 